\title{
Identification of Three Novel Plasmodium Factors Involved in Ookinete to Oocyst Developmental Transition
}

\author{
Chiamaka V. Ukegbu ${ }^{1}$, George K. Christophides ${ }^{1,2}$ and Dina Vlachou ${ }^{1,2 *}$ \\ ${ }_{1}^{1}$ Department of Life Sciences, Imperial College London, London, United Kingdom, ${ }^{2}$ The Cyprus Institute, Nicosia, Cyprus
}

\section{OPEN ACCESS}

Edited by:

Chandra Ramakrishnan,

University of Zurich, Switzerland

Reviewed by:

Katarzyna Kinga Modrzynska,

University of Glasgow,

United Kingdom

Rita Tewari,

University of Nottingham,

United Kingdom

*Correspondence:

Dina Vlachou

d.vlachou@imperial.ac.uk

Specialty section:

This article was submitted to

Parasite and Host,

a section of the journal

Frontiers in Cellular

and Infection Microbiology

Received: 27 November 2020

Accepted: 10 February 2021

Published: 15 March 2021

Citation:

Ukegbu CV, Christophides GK and Vlachou D (2021) Identification of

Three Novel Plasmodium Factors

Involved in Ookinete to Oocyst

Developmental Transition.

Front. Cell. Infect. Microbiol. 11:634273.

doi: 10.3389/fcimb.2021.634273
Plasmodium falciparum malaria remains a major cause of global morbidity and mortality, mainly in sub-Saharan Africa. The numbers of new malaria cases and deaths have been stable in the last years despite intense efforts for disease elimination, highlighting the need for new approaches to stop disease transmission. Further understanding of the parasite transmission biology could provide a framework for the development of such approaches. We phenotypically and functionally characterized three novel genes, PIMMS01, PIMMS57, and PIMMS22, using targeted disruption of their orthologs in the rodent parasite Plasmodium berghei. PIMMS01 and PIMMS57 are specifically and highly expressed in ookinetes, while PIMMS22 transcription starts already in gametocytes and peaks in sporozoites. All three genes show strong phenotypes associated with the ookinete to oocyst transition, as their disruption leads to very low numbers of oocysts and complete abolishment of transmission. PIMMS22 has a secondary essential function in the oocyst. Our results enrich the molecular understanding of the parasite-vector interactions and identify PIMMS01, PIMMS57, and PIMMS22 as new targets of transmission blocking interventions.

Keywords: ookinete development, malaria transmission, ookinete to oocyst transition, mosquito midgut invasion, Plasmodium sporogonic development, vector-parasite interactions

\section{INTRODUCTION}

Malaria remains a global public health problem with 228 million cases in 2018, causing 405,000 deaths (WHO World Malaria Report 2019). This deadly disease is caused by parasites of the genus Plasmodium transmitted by the bite of infected female Anopheles mosquitoes. P. falciparum is the most virulent of the human malaria parasites, responsible for most of malaria-associated deaths especially of children under 5 years old and first-time pregnant women. Transmission begins upon ingestion by a female Anopheles mosquito of a blood meal containing male and female gametocytes from an infected human. In the mosquito midgut, the gametocytes differentiate into male and female gametes that then mate to form the zygote. Within $24 \mathrm{~h}$, the zygote, through several stages of development, differentiates into a crescent shaped motile ookinete that escapes the blood bolus and its encasing peritrophic matrix and invades and traverses the midgut epithelium. On the basal side of the midgut, the ookinete transforms into a sessile, replicative oocyst where thousands of 
sporozoites are produced. Oocysts burst and sporozoites released into the haemocoel reach the salivary glands from where they are transmitted to a new host during a subsequent mosquito bite.

The ookinete-to-oocyst developmental transition in the mosquito midgut is the most critical stage of the entire parasite transmission cycle. The ingested parasite populations suffer major losses during this stage resulting in very few oocysts and, in most cases, termination of transmission. This stage is therefore a good target of interventions aiming to control disease transmission (Smith et al., 2014). Genome scale transcriptomic studies have shed important insights into Plasmodium gene expression and regulation that accompanies developmental stage transitions and developmental processes of the parasite (Le Roch et al., 2003; Hall et al., 2005; Otto et al., 2010; Otto et al., 2014; Lasonder et al., 2016; Yeoh et al., 2017; Reid et al., 2018). We have previously used DNA microarrays to identify transcriptional profiles driving the under characterized gametocyte-ookinete-to-oocyst developmental transitions in the rodent model parasite Plasmodium berghei (Akinosoglou et al., 2015); and more recently single cell RNA sequencing in $P$. berghei has also revealed genes differentially expressed during ookinete-to-oocyst developmental transition (Howick et al., 2019).

While rodent models are routinely employed in study parasite development in the mosquito vector, due to their genetic accessibility and tractability, functional differences in the utilization of genes between rodent and human parasites may be important especially with regards to specific interactions with the vector (Dong et al., 2006; Simões et al., 2017). Indeed, to cope with the live and changing host environment, Plasmodium has evolved the ability to undertake transcriptional variation for its survival and transmission (Rovira-Graells et al., 2012; Waters, 2016). P. falciparum utilizes and infects about 60-70 Anopheles species, therefore, there would be a necessity to adapt to the different environments of each of these mosquito species, in particular with regards to differences in mosquito immune responses, behavior, and physiology (White et al., 2011; Mitri et al., 2015; Costa et al., 2018; Lefevre et al., 2018). For these reasons, we have developed an operational framework where $P$. falciparum genes potentially involved in parasite interactions with the vector are identified using genome-wide transcriptomic studies in near field settings and then prioritized for genetic and functional characterization in laboratory mosquito infections with $P$. berghei.

Here, we selected from our dataset three $P$. falciparum genes that exhibit highly abundant transcripts $24 \mathrm{~h}$ post blood feeding (hpbf) in the midguts of Anopheles coluzzii mosquitoes. These genes have been part of a large reverse genetic $P$. berghei screen to identify genes that function during parasite infection of the mosquito midgut, designated as Plasmodium Infection of the Mosquito Midgut Screen (PIMMS). We have previously reported the characterization of PIMMS2 that encodes an ookinete membrane-associated subtilisin-like protein involved in midgut traversal (Ukegbu et al., 2017) and PIMMS43 that encodes another ookinete membrane protein aiding in resistance to responses of the mosquito complement-like system (Ukegbu et al., 2020). Now we report the identification and characterization of three additional genes, PIMMS01, PIMMS57, and PIMMS22, which are highly transcribed and translated in the ookinete; with PIMMS22 also highly expressed at both the gametocyte and sporozoite stages. PIMMS01 and PIMMS57 are putatively secreted and membrane-bound proteins, respectively, while PIMMS22 does not include any known secretory or membrane association signals but localizes on the periphery of ookinetes and sporozoites, possibly the inner membrane complex. An earlier study by Zheng and colleagues designated PIMMS57 as PSOP26 (Zheng et al., 2016) and showed the antibodies against $P$. berghei PIMMS57 can affect ookinete maturation and malaria transmission. We demonstrate that none of these genes has a role in ookinete development but are all important for the ookinete-to-oocyst developmental transition in the midgut and disease transmission.

\section{MATERIALS AND METHODS}

\section{Ethics Statement}

Animal procedures were carried out in accordance with the Animal (Scientifics Procedures) Act 1986 under the UK Home Office Licenses PPL70/8788.

\section{Sequence Analysis}

Plasmodium protein sequences were retrieved from PlasmoDB (http://plasmodb.org/plasmo/) and apicomplexan parasite sequences were retrieved from UniProt. Alignment was carried out using Clustal Omega and visualized in the BioEdit sequence alignment editor program. Signal peptide and transmembrane domains were predicted using SignalP and Phobius (Käll et al., 2007; Armenteros et al., 2019).

\section{$P$. falciparum Culturing and Mosquito Infections}

P. falciparum NF54 was cultured as described previously (Habtewold et al., 2019). Briefly, ABS and gametocytes were cultured using human RBCs of various blood groups in the following order of preference: $\mathrm{O}+$ male, $\mathrm{O}+$ female, $\mathrm{A}+$ male, and $\mathrm{A}+$ female. Asexual cultures were set up in $10 \mathrm{ml}$ complete medium [RPMI-1640, 0.05 g/L Hypoxanthine, $0.3 \mathrm{mg} / \mathrm{L} \mathrm{L-}$ glutamine, $10 \%(\mathrm{v} / \mathrm{v})$ sterile human serum of A+ serotype] containing a final volume of $500 \mu \mathrm{l}$ of hRBCs $(0.3 \%-4 \%$ infection). Cultures were gassed with "malaria gas" $\left(3 \% \mathrm{O}_{2} / 5 \%\right.$ $\mathrm{CO}_{2} / 92 \% \mathrm{~N}_{2}$ ) and incubated at $37^{\circ} \mathrm{C}$. Gametocyte cultures were set up by dilution of a 3-4\% ring stage asexual culture to $1 \%$ ring forms in a final volume of $8 \mathrm{ml}$ complete medium and supply of fresh hRBCs. The cultures underwent daily exchange of around $75 \%$ of the media, gassed with malaria gas and incubated at $37^{\circ} \mathrm{C}$ until day 14. Mosquitos were infected with P. falciparum by SMFAs as described previously (Habtewold et al., 2019). Briefly, Giemsa staining was used to assess gametocyte density and in vitro exflagellation was used to assess viability of stage $\mathrm{V}$ male 
gametocytes. In a pre-warmed tube, the gametocyte cultures were pooled in a final volume of $300 \mu \mathrm{l}$ containing $20 \%(\mathrm{v} / \mathrm{v})$ uninfected serum-free hRBCs and 50\% (v/v) heat-inactivated human serum. This mixture was then transferred to pre-warmed mosquito feeders kept a constant temperature of $37^{\circ} \mathrm{C}$.

\section{$\boldsymbol{P}$. berghei Strains and Cultivation}

$P$. berghei lines used were the reference parent line of $P$. berghei ANKA $2.34(c l 15 c y 1)$ and the $507 m 6 c l 1$ (c507) line that contains GFP integrated into the 230p gene locus (PBANKA_0306000) without a drug selectable marker and constitutively expresses GFP under the control of the EF1 alpha promoter (Janse et al., 2006a). All parasite lines were maintained in 8-10-week-old CD1 and/or TO female mice by serial passaging. P. berghei mixed blood stages, gametocytes, and ookinetes were cultured and purified as described previously (Janse and Waters, 1995; Beetsma et al., 1998).

\section{Quantitative RT-PCR}

Total RNA was extracted from $P$. berghei and $P$. falciparum parasites or $A$. coluzzii infected with $P$. berghei or $P$. falciparum using Trizol reagent (ThermoFisher) according to the manufacturer's instructions. cDNA was synthesized using the PrimeScript Reverse Transcription Kit (Takara) after Turbo DNase (ThermoFisher) treatment. For qRT-PCR, SYBR green (Takara) and gene specific qRT-PCR primers (Table S6) were used according to the manufacturer's guidelines. Gene expression was normalized against GFP in $P$. berghei and against $P$. falciparum arginyl-tRNA synthetase in $P$. falciparum using the $\Delta \Delta \mathrm{Ct}$ method.

\section{Antibody Production}

Rabbit polyclonal antibodies against peptides of the deduced proteins PIMMS01 and PIMMS57 were raised and purified from the serum of an immunized rabbit (Eurogentec): $\alpha$-Pfc01 targets the PfPIMMS01 peptide EKHKDSTKWDKSYSF (aa 72-86); $\alpha$ Pbc57 targets the PbPIMMS57 the N-terminal peptide SNDSNYEDRDNAPNR (aa 48-62); and $\alpha$-Pfc57, targets the N-terminal peptide EQRVRDEGRENNRRS (aa 111-125.)

\section{Western Blot Analysis}

Soluble cell lysates were prepared by suspending purified parasite pellets in cell lysis buffer ( $5 \mathrm{mM}$ Tris, $150 \mathrm{mM} \mathrm{NaCl}$ ) containing protease inhibitors. Triton X-100 soluble cell lysates were prepared by suspending purified parasite pellets in Triton X100 cell lysis buffer ( $5 \mathrm{mM}$ Tris, $150 \mathrm{mM} \mathrm{NaCl}, 1 \%$ v/v Triton X100) containing protease inhibitors. Triton X-100 insoluble cell fractions/whole cell lysates were prepared by suspending parasite pellets or infected midguts in reducing $(3 \% \mathrm{v} / \mathrm{v} \mathrm{2-}$ mercapthoethanol) Laemmli buffer. Protein samples were then boiled under reducing (3\% v/v 2-mercapthoethanol) conditions in Laemmli buffer and separated using sodium dodecyl sulfate polyacrylamide gel electrophoresis. Separated proteins were then transferred to a PVDF membrane (GE Healthcare). Proteins were detected using Goat $\alpha$-GFP (Rockland chemicals) (1:100), mouse $\alpha$-tubulin (Sigma), and 3D11 mouse monoclonal $\alpha$ PbCSP (Potocnjak et al., 1980) (1:1000), $\alpha$-Pbc57 (1:100), $\alpha$ -
Pfc01 (1:50), $\alpha$-Pfc57 (1:50), and $\alpha$-Pfs25 (Barr et al., 1991), (1:100) antibodies. Secondary horseradish peroxidase (HRP) conjugated IgG, goat $\alpha$-mouse IgG antibodies (Promega), and donkey $\alpha$-goat IgG (Abcam) were used at 1: 10,000 and 1: 5,000 dilutions, respectively. All primary and secondary antibodies were diluted in $3 \%$ milk-PBS-Tween $(0.05 \% \mathrm{v} / \mathrm{v})$ blocking buffer.

\section{Indirect Immunofluorescence Assay}

For IFAs on blood bolus parasites, the blood bolus was collected from dissected midguts of mosquitoes at 2 and 19 hpbf. The blood bolus was washed in PBS and fixed in $4 \%$ paraformaldehyde (PFA) for $10 \mathrm{~min}$. The fixed parasites were smeared on a glass slide, permeabilized with $0.2 \%(\mathrm{v} / \mathrm{v})$ Triton X100 , and blocked with $3 \%(\mathrm{w} / \mathrm{v})$ bovine serum albumin Purified gametocytes and in vitro ookinetes were fixed, permeabilized and blocked as above. For IFAs on midgut sporozoites at $15 \mathrm{dpbf}$, infected midguts were dissected, and tissues were homogenized to release sporozoites. Sporozoites were fixed, blocked and permeabilized as that above. For IFAs on ookinetes invading the midgut epithelium, the midguts of mosquitoes at $26 \mathrm{hpbf}$ were dissected, and the blood boluses were discarded. The midgut epithelium was fixed in 4\% PFA in PBS for 45 min and washed thrice in PBS for $10 \mathrm{~min}$ each. Midgut epithelium was permeabilized and blocked for $1 \mathrm{~h}$ in $1 \% \mathrm{w} / \mathrm{v}$ BSA, $0.1 \% \mathrm{v} / \mathrm{v}$ Triton X-100 in PBS blocking solution. Samples were then stained in blocking solution with primary antibodies $(\alpha-G F P$, $1: 100 ; 13.1$ mouse monoclonal $\alpha-\mathrm{P} 28$ (Winger et al., 1988), $1: 1000$; and $\alpha$-PbCSP $1: 100 ; \alpha$-Pfc01, $1: 100 ; \alpha$-Pfc57, $1: 100$; $4 \mathrm{~B} 7$ mouse monoclonal $\alpha$-Pfs25, 1:100. Alexa Fluor (488 and 568 ) conjugated secondary antibodies specific to goat or mouse (ThermoFisher) were used at a dilution of 1:1000. 4',6diamidino-2-phenylindole (DAPI) was used to stain nuclear DNA. Images were acquired using a Leica SP5 MP confocal laser-scanning microscope. Images underwent processing by deconvolution using Huygens software and were visualized using Image J.

\section{Generation of Transgenic Parasites}

For GFP tagging of $\mathrm{Pbc01}$ in the 2.34 line, a $603 \mathrm{bp}$ ApaI/HindIII 5' and a 770 bp EcoRI/BamHI 3' homology arm region were amplified from $P$. berghei 2.34 genomic DNA using the primer pairs $\mathrm{P} 1 / \mathrm{P} 2$ and $\mathrm{P} 3 / \mathrm{P} 4$, respectively. For GFP tagging of $\mathrm{Pbc57}$ in the 2.34 line, a 919 bp ApaI/SacII 5' homology arm corresponding to the most 3 ' region of the CDS without the stop codon and a $359 \mathrm{bp}$ XhoI/XmaI 3' homology arm region corresponding to the $3^{\prime}$ UTR of the gene were amplified using the primer pairs P5/P6 and P7/P8, respectively. For GFP tagging of $\mathrm{Pbc22}$ in the 2.34 line, a $753 \mathrm{bp} \mathrm{ApaI/HindIII} \mathrm{5'} \mathrm{homology} \mathrm{arm}$ corresponding to the most 3' region of the CDS without the stop codon and a 530 bp EcoRI/BamHI 3' homology arm region corresponding to the 3' UTR of the gene were amplified using the primer pairs $\mathrm{P} 9 / \mathrm{P} 10$ and $\mathrm{P} 11 / \mathrm{P} 12$, respectively. The $\mathrm{Pbc01}$ and $P b c 22$ fragments were cloned into the pBS-TgDHFR vector which carries a modified Toxoplasma gondii dihydrofolate gene (TgDHFR/TS) cassette that confers resistance to pyrimethamine (Dessens et al., 1999). The Pbc57 fragments were cloned into plasmid pL0035 which carries the hDHFR selection cassette 
(Braks et al., 2006). Finally, to put GFP tag in frame with the 3' region of the CDS, a HindIII or SacII GFP-P. berghei DHFR 3'UTR fragment was amplified from the pL00018 vector (MRA787, MR4) using primers $\mathrm{P} 13 / \mathrm{P} 14$ or $\mathrm{P} 15 / \mathrm{P} 16$, respectively.

For $3 X H A$ tagging of $\mathrm{Pbc01}$ in the c507 line, a $687 \mathrm{bp} \mathrm{ApaI/}$ SacII 5' homology arm corresponding to the some of the 5'UTR, the whole CDS without the stop codon and the 3XHA tag and a $2051 \mathrm{bp} \mathrm{XhoI/XmaI} \mathrm{3'} \mathrm{homology} \mathrm{arm} \mathrm{region} \mathrm{corresponding} \mathrm{to}$ the 3' UTR of the gene was amplified from genomic DNA using the Gibson assembly primer pairs P43/P44 and P45/P46, respectively. For $3 X H A$ tagging of $\mathrm{Pbc57}$ in the c507 line, a 1003 bp ApaI/SacII 5' homology arm corresponding to the most 3 ' region of the CDS without the stop codon and the 3XHA tag and a $359 \mathrm{bp}$ XhoI/XmaI 3' homology arm region corresponding to the 3' UTR of the gene was amplified using the Gibson assembly primer pairs $\mathrm{P} 47 / \mathrm{P} 48$ and $\mathrm{P} 49 / \mathrm{P} 50$ respectively. The $\mathrm{Pbc01}$ and $\mathrm{Pbc57}$ fragments were cloned into plasmid pL0035 via Gibson assembly.

For partial disruption of $\mathrm{Pbc01}$ in the $c 507$ line, a $432 \mathrm{bp} \mathrm{ApaI/}$ HindIII 5' homology arm and a 1102 bp EcoRI/BamHI 3' homology arm was amplified using the primer pairs P24/P25 and P26/P27, respectively. For partial disruption of $P b c 57$ in the c507 line, a 590 bp ApaI/HindIII 5' homology arm and a 684 bp EcoRI/BamHI 3' homology arm was amplified using the primer pairs P28/P29 and P30/P31, respectively. For partial disruption of $\mathrm{Pbc22}$ in the $c 507$ line, a $560 \mathrm{bp}$ ApaI/HindIII 5' homology arm and a 712 bp EcoRI/BamHI 3' homology arm was amplified using the primer pairs $\mathrm{P} 32 / \mathrm{P} 33$ and $\mathrm{P} 34 / \mathrm{P} 35$, respectively. These fragments were cloned into the $\mathrm{pBS}$-TgDHFR vector. The targeting cassettes by ApaI/BamHI digestion allows knockout of $39 \%$ of $P b c 01$ CDS, $70 \%$ of $P b c 57$ CDS, and $80 \%$ of $P b c 22$ CDS.

Transfection of linearized constructs, selection of transgenic parasites and clonal selection was carried out as described previously (Janse et al., 2006b).

\section{Genotypic Analysis of Transgenic Parasites}

Blood stage parasites were purified by removal of white blood cells using hand packed cellulose (Sigma) columns. Parasites were released using red blood cell lysis buffer $\left(0.17 \mathrm{M} \mathrm{NH}_{4} \mathrm{Cl}\right)$ on ice for $20 \mathrm{~min}$. Genomic DNA was extracted from parasites using the DNeasy kit (Qiagen). Integration events or maintenance of the unmodified locus was detected by PCR on genomic DNA using primers listed in Table S6. For PFGE, blood stage parasites within agarose plugs were lysed in lysis buffer (1X TNE, $0.1 \mathrm{M}$ EDTA pH 8.0, 2\% (v/v) Sarkosyl, $400 \mu \mathrm{g} / \mathrm{ml}$ proteinase $\mathrm{K}$ ) to release nuclear chromosomes. PFGE separated chromosomes (Run settings: 98 volts, $1-5$ mins pulse time for $60 \mathrm{~h}$ at $14^{\circ} \mathrm{C}$ ) were then subjected to Southern blot analysis using a probe targeting the TgDHFR/TS-P. berghei DHFR 3'UTR, obtained by HindIII and EcoRV digestion of the pBS-TgDHFR plasmid.

\section{Phenotypic Assays}

Exflagellation assays were performed as described previously (Akinosoglou et al., 2015). Briefly, blood from a high gametocytemic mouse was added in a 1:40 ratio to ookinete medium (RPMI 1640, 20\% v/v FBS, $100 \mu \mathrm{M}$ xanthurenic acid, $\mathrm{pH}$ 7.4), and exflagellation was counted in a standard hemocytometer under a light microscope. Conversion assays were performed as previously described (Akinosoglou et al., 2015). Briefly, in vitro cultivated ookinetes were resuspended in $50 \mu \mathrm{l}$ of fresh ookinete and incubated with a Cy3-labeled 13.1 mouse monoclonal $\alpha$-P28 (1:50 dilution) for $20 \mathrm{~min}$ on ice. The conversion rate was calculated as the percentage of $\mathrm{Cy} 3$ positive ookinetes to $\mathrm{Cy} 3$ positive macrogametes and ookinetes.

Ookinete motility assays were performed as described previously (Moon et al., 2009). Briefly, on a glass slide, $24 \mathrm{~h}$ in vitro ookinete culture was incubated with Matrigel (BD biosciences), and allowed to set at RT for $30 \mathrm{~min}$. On a Leica DMR fluorescence microscope and a Zeiss Axiocam HRc camera controlled by the AxioVision (Zeiss) software, time-lapse images ( 1 frame every $5 \mathrm{~s}$ for $10 \mathrm{~min}$ ) of ookinetes were obtained. Using the manual tracking plugin in the Icy software (http://icy. bioimageanalysis.org/), the speed of individual ookinetes was measured.

A. coluzzii mosquitoes were fed by direct blood feeding as previously described (Sinden, 1997) on mice with parasitemia of $4 \%-5 \%$ and gametocytemia of $1 \%-2 \%$. Midguts tissues were dissected at 7-10 dpbf and fixed in 4\% PFA in PBS and mounted in Vectashield ${ }^{\circledR}$ (VectorLabs). Oocysts or melanized ookinetes were counted using light and/or fluorescence microscopy. 2530 P. berghei infected A. coluzzii midguts or salivary glands at 15 and 21 dpbf respectively were homogenized and oocyst and salivary gland sporozoites counted using a standard hemocytometer. Finally, in mosquito to mouse transmission assays, at least $30 \mathrm{P}$. berghei infected mosquitoes at $21 \mathrm{dpbf}$ were allowed to feed on two to three anaesthetized C57/BL6 mice for $15 \mathrm{~min}$. Parasitemia was monitored until 14 days post mosquito bite by Giemsa staining of blood smears.

\section{Ookinete Injections in Mosquito Haemocoel}

Ookinete injections were carried out as described previously (Bushell et al., 2009). Briefly, the concentration of ookinetes from $24 \mathrm{~h}$ in vitro cultures was adjusted with RPMI 1640, and this was injected using glass capillary needles and the Nanoject II microinjector (Drummond Scientific) into the thorax of $A$. coluzzii mosquitoes at a final concentration of 800 ookinetes per mosquito.

\section{Gene Silencing}

Total RNA extracted from $A$. coluzzii midgut infected with $P$. berghei c507 at $24 \mathrm{hpbf}$ was used to prepare cDNA. The cDNA was used in conjunction with primers reported in (Habtewold et al., 2008) to amplify CTL4. DsRNA was then produced using the resulting PCR product and the T7 high yield transcription kit (ThermoFisher). $0.2 \mu \mathrm{g}$ of purified dsRNA in $69 \mathrm{nl}$ was injected into the thorax of $A$. coluzzii mosquitoes using glass capillary needles and the Nanoject II microinjector. Two to three days post injected mosquitoes were then infected with $P$. berghei. 


\section{Statistical Analysis}

Statistical analyses were performed using GraphPad Prism v8.0. Statistical analyses for exflagellation, ookinete conversion and motility assays were performed using a two-tailed, unpaired Student's t-test. For statistical analyses of the oocyst or melanized parasite load, P-values were calculated using the Mann-Whitney test.

\section{RESULTS}

\section{In Silico Analysis}

Based on their expression profiles in a $P$. falciparum transcriptomics dataset and mutant phenotypes in a $P$. berghei high throughput reverse genetics screen, three parasite genes were selected for further characterization, including targeted disruption and detail phenotypic analysis in $P$. berghei. These genes were PF3D7_0112100 in P. falciparum and PBANKA_0201700 in P. berghei, PF3D7_1244500 in $P$. falciparum and PBANKA_1457700 in $P$. berghei, and PF3D7_0814600 in P. falciparum and PBANKA_1422900 in $P$. berghei; designated as PIMMS01, PIMMS57, and PIMMS22, respectively.

PfPIMMS01 encodes a 163 amino acid-long protein $(19 \mathrm{kDa})$ with a predicted signal peptide (aa 1-27; probability according to SignalP and Phobius 0.688 and 0.904 , respectively). Its $P$. berghei orthologue, PbPIMMS01, encodes a much shorter 85 amino acid-long protein $(9 \mathrm{kDa})$ and also contains a predicted signal peptide (aa 1-26; probability according to SignalP and Phobius 0.826 and 0.978 , respectively). This suggests that both proteins are putatively secreted. While the central part of the deduced protein is highly conserved between all PIMMS01 orthologues, all rodent PIMMS01 proteins are shorter than their orthologues in human parasites, lacking the entire second half of the protein (Figure S1). InterPro domain analysis revealed no recognizable domain, and BLAST searches showed that the protein is Plasmodium specific.

PfPIMMS57 encodes a 810 amino acid-long $(94 \mathrm{kDa})$ protein with a predicted signal peptide (aa 1-26; probability according to SignalP and Phobius 0.697 and 0.657 , respectively) that overlaps with a putative transmembrane domain albeit with low probability according to Phobius (0.343). A second transmembrane domain close to the carboxy terminus of PfPIMMS57 (aa 677-697) is predicted with very high probability (0.874). The $P$. berghei orthologue, PbPIMMS57, encodes a protein of 774 amino acid-long $(90 \mathrm{kDa})$ protein with two putative transmembrane domains (aa 6-23 and aa 633653) predicted with high probability (0.984 and 0.967 , respectively). These data suggest that both proteins are putatively membrane-bound. Higher sequence conservation between PIMMS57 orthologues is observed in the second half of the proteins compared to the first half, pointing to a conserved functional role served by this region (Figure S2). A previous in silico analysis identified in PfPIMMS57, two P. falciparum serine/ threonine protein phosphatase type I catalytic (PfPP1c) binding motifs, the RVxF motif, RRKVNF (aa 347-352) and the Fxx[RK] $\mathrm{x}$ [RK] motif, FNKILKR (aa 488-494) (Hollin et al., 2016). These motifs are not conserved in the other PIMMS57 orthologues.

Finally, PfPIMMS22 encodes a 393 amino acid (45 kDa) protein and PbPIMMS22 encodes a 393 aa $(44 \mathrm{kDa})$ protein, both with no predicted signal peptide or transmembrane domain. The protein is highly conserved amongst Plasmodium orthologues with sequence identity ranging from $96 \%$ in PyPIMMS22 to $76 \%$ and $80 \%$ in PfPIMMS 22 and PvPIMMS22, respectively (Figure S3). PyPIMMS22 (PY17X_1424900) was previously identified in salivary gland sporozoites through a subtractive hybridization ( $\mathrm{SSH}$ ) profiling and termed sporozoite protein S15 (Kaiser et al., 2004). The same protein was also identified in midgut oocyst sporozoites as an interacting partner to the apicomplexan specific RNA-binding protein, ALBA4, which is involved in mRNA regulation in gametocyte and midgut oocyst sporozoite development (Muñoz et al., 2017). PIMMS22 homologues are found in other apicomplexan parasites including Toxoplasma gondii, Neospora caninum and Eimeria with sequence identities to PIMMS22 ranging from $36 \%$ to $42 \%$ (Figure S4). InterPro domain analysis revealed no recognizable domain in PIMMS22.

\section{Transcription Profiles}

We searched a DNA microarray dataset of $A$. coluzzii midguts infected with $P$. falciparum field isolates from Burkina Faso to examine the transcriptional profiles of the three genes under study. PfPIMMS01 and, to a lesser extent, PfPIMMS57 were lowly transcribed $1 \mathrm{~h}$ post blood-feeding (hpbf) and their transcription peaked 24 hpbf, while PfPIMMS22 expression started in gametocytes and continued at high levels $24 \mathrm{hpbf}$. We corroborated these data with quantitative real-time RT-PCR (qRT-PCR) in laboratory P. falciparum NF54 cultured gametocytes and in NF54 infections of A. coluzzii 1 and 24 hpbf (Figure 1A). Very low levels of PfPIMMSO1 and PfPIMMS57 transcripts were detected in gametocytes and in A. coluzzii midguts $1 \mathrm{hpbf}$, peaking at $24 \mathrm{hpbf}$.

Transcription of the $P$. berghei orthologous genes was also analyzed using qRT-PCR (Figure 1B). In this assay, the $P$. berghei line ANKA507m6cl1 that constitutively expresses GFP was used (Janse et al., 2006a); hereafter referred to as c507. PbPIMMS01 and PbPIMMS57 transcripts were highly abundant in purified mature ookinetes (Ook) and not detectable in mixed blood stages (MBS) and purified gametocytes. While PbPIMMS57 appears to be specific for ookinetes, low levels of PbPIMMS01 transcripts are also detected in mature oocysts and midgut sporozoites at 10 days and 15 days post blood-feeding (dpbf). Like PfPIMMS22, expression of PbPIMMS22 starts in gametocytes and continues at high levels $24 \mathrm{hpbf}$ and peaks in midgut sporozoites $15 \mathrm{dpbf}$ (Figure 1B). Low abundance PbPIMMS22 transcripts detected in MBS is likely due to expression in gametocytes. These results generally agree with those in published RNA-sequencing data from blood stages and ookinetes (Otto et al., 2014; Yeoh et al., 2017).

\section{Protein Expression and Localization}

Protein expression was assessed by endogenous GFP tagging of the three $P$. berghei genes via double crossover homologous 

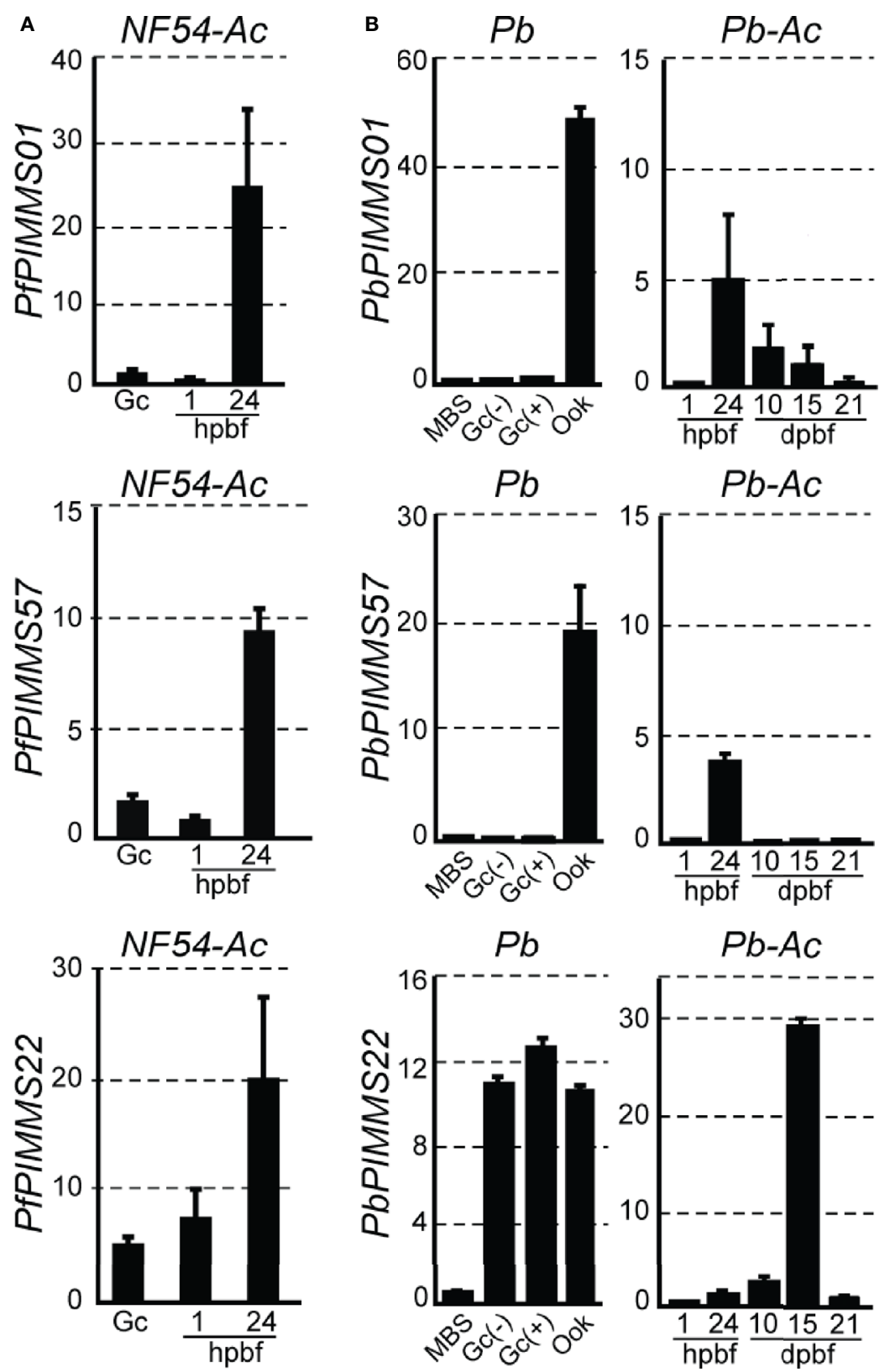

FIGURE 1 | PIMMS01, PIMMS57, and PIMMS22 transcriptional profiles. (A) Relative abundance of PfPIMMS01, PfPIMMS57, and PfPIMMS22 transcripts in purified $P$. falciparum in vitro cultured gametocytes, and $A$. coluzzii mosquito infected midguts at 1 and 24 hpbf, as determined by qRT-PCR in NF54 parasite populations and normalized against the Arginyl-tRNA synthetase. (B) Relative abundance of $P$. berghei PIMMS01, PbPIMMS57, and PbPIMMS22 transcripts in purified blood stages and in vitro produced ookinetes, and in A. coluzzii mosquito stages, as determined by qRT-PCR in the c507 line and normalized against the constitutive expressed GFP. In all panels. each bar is the average of three biological replicates. Error bars indicate SEM. MBS, mixed blood stages; Gc, gametocytes; Gc(+), activated gametocytes; Gc(-), non-activated gametocytes; Ook, ookinetes; hpbf, hours post blood feeding; dpbf, days post blood feeding.

recombination in the ANKA 2.34 line. The generated transgenic parasites were named $c 01:: g f p, c 57:: g f p$, and $c 22:: g f p$ (Figure S5). P. berghei PIMMS01 and PIMMS57 genes were also terminally tagged with the $3 \mathrm{xHA}$ (hemagglutinin) tag in the $c 507$ line, and transgenic parasites were named $c 01:: 3 x h a$ and $c 57:: 3 x h a$ (Figure S6). We also generated rabbit polyclonal antibodies raised against peptides of the deduced proteins in both $P$. berghei and
$P$. falciparum. Of all antibodies, those that produced data and are analyzed below were: $\alpha$-Pfc01, PfPIMMS01 peptide aa 72-86; $\alpha$ Pbc57, PbPIMMS57 peptide aa 48-62; and $\alpha$-Pfc57, PfPIMMS57 peptide aa 111-125.

Mosquito stage development of transgenic GFP-tagged $P$. berghei lines was assessed by counting midgut sporozoite numbers 15 dpbf. Amongst the three transgenic parasites, only 
c22::gfp produced wild type (wt) number of midgut sporozoites (Table S1). Lines $c 01:: g f p$ and $c 57:: g f p$ yielded severely reduced sporozoite numbers, suggesting that insertion of GFP compromises the function of the two proteins. Similar to their GFP-tagged counterpart and concomitant gene knockout (see next section) parasite lines, both HA-tagged transgenic lines, showed severely reduced oocyst numbers compared to the c507 reference parasite, as assessed by counting oocyst numbers 8 dpbf (Table S2). This phenotype suggests that insertion of the $3 \mathrm{xHA}$ tag compromises the function of the two proteins.

\section{PIMMS01 Expression and Localization}

Western blot analysis using an $\alpha$-GFP antibody on the nonfunctional c01::gfp line confirmed high levels of PbPIMMS01:: GFP fusion protein at the expected molecular weight (Figure 2A). The protein was found to be highly expressed in mature ookinetes of the c01::gfp line and be absent from MBS or gametocytes or any stage of the ANKA 2.34 background parasite. Similarly, western blot analysis on the c01::3xha transgenic line using an $\alpha-\mathrm{HA}$ antibody confirmed expression of the PbPIMMS01::3xHA fusion protein in the ookinete at the expected molecular weights and absence from gametocytes (Figure 2B). The protein was only detected in the Triton X100 soluble fraction suggesting that it is not membrane associated.

Despite the strong evidence that the PbPIMMS01 GFP- and HA-tagged proteins are non-functional, these are still likely to be localised correctly. IFAs on the developmentally compromised c01::gfp and c01::3xha transgenic lines revealed strong cytoplasmic localization of both PbPIMMS01::GFP and PbPIMMS01::3xHA (Figure 2C), which indicates that the protein may be stuck in a secretory pathway and suggests that the native polypeptide may be associated with the ookinete outer membrane especially during invasion. Peptide antibodies raised against $P$. berghei PIMMS01 did not work in western blots and IFAs.
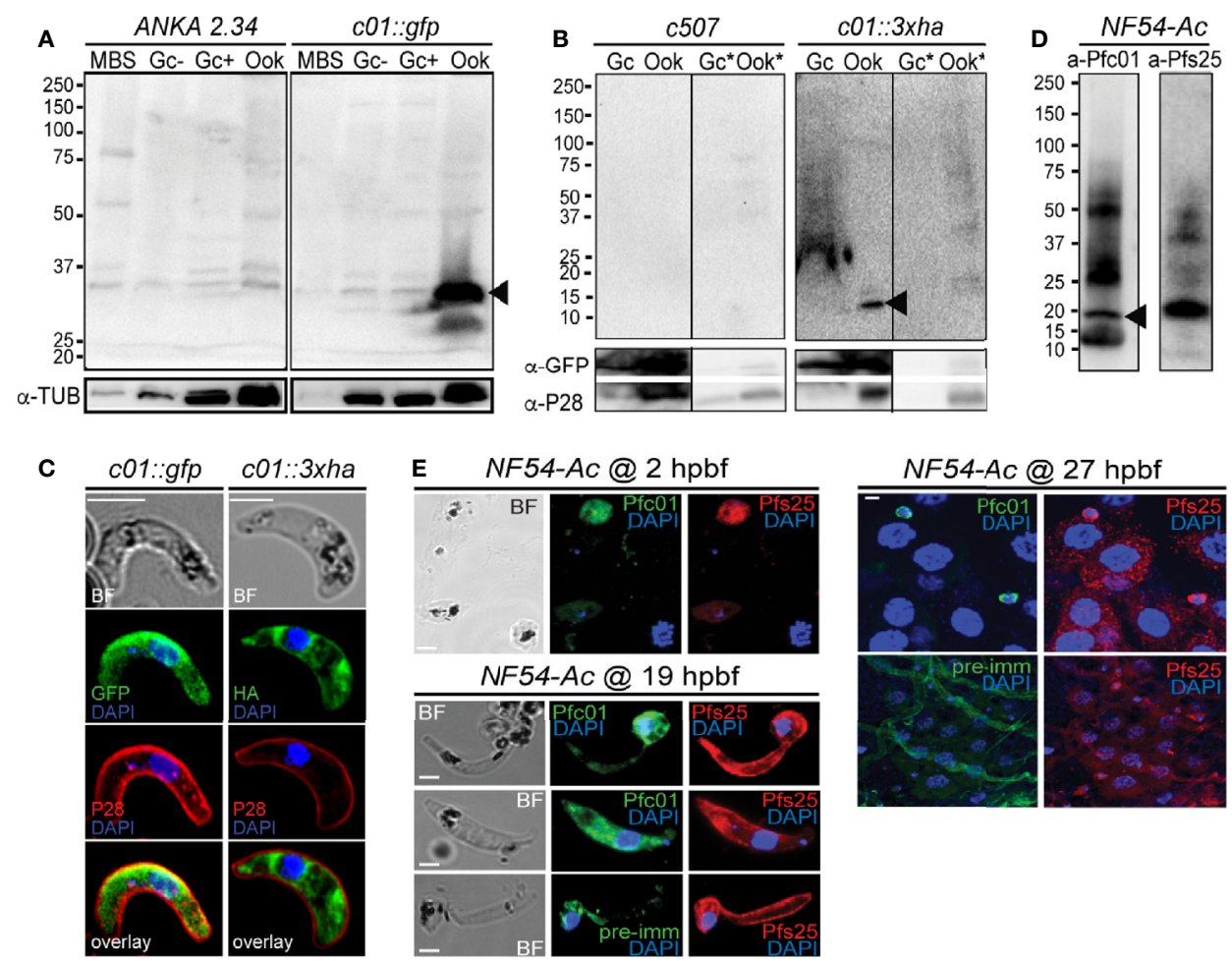

FIGURE 2 | PIMMS01 protein expression and localization. (A) Western blot analysis using $\alpha$-GFP antibody on whole cell lysates of $P$. berghei c01::gfp parasites. The PbPIMMS01::GFP fusion protein band is indicated with a black arrowhead. The ANKA 2.34 parental parasite line was used as a negative control. Tubulin was used as a loading control. MBS, mixed blood stages; Gc(-), non-activated gametocytes; Gc(+), activated gametocytes; Ook, ookinetes. (B) Western blot analysis using the $\alpha-\mathrm{HA}$ antibody on Triton X-100 soluble lysates of in vitro gametocyte (Gc) and ookinetes (Ook) and Triton X-100 insoluble lysates (Gc*, Ook ) of $c 01:: 3 \times$ ha. The $\mathrm{c} 507$ reference line (left panel) was used as a negative control. PbPIMMS01::HA fusion protein band is indicated with a black arrowhead. GFP and P28 were used as a loading and a stage specific control, respectively. (C) Immunofluorescence assays on ookinetes of PIMMS01 tagged with GFP (left panel) and 3xHA (right panel) stained with $\alpha$-GFP or $\alpha$-HA (green), respectively, as well as with the ookinete surface $\alpha$-P28 (red). DNA was stained with DAPI. Images are de-convoluted projections of confocal stacks. BF, bright field; Scale bars, $5 \mu \mathrm{m}$. (D) Western blot analysis using $\alpha$-Pfc01 antibody on whole cell lysates of coluzzii midguts at $22 \mathrm{~h}$ post blood feeding (hpbf). The PfPIMMS01 protein band is indicated with a black arrowhead. Tubulin was used a loading control for Gc and Sch while Pfs25 was used for 22 hpbf. (E) Immunofluorescence assays of $P$. falciparum NF54 parasites in mosquito blood bolus at 2 hpbf, ookinetes in mosquito blood bolus at 19 hpbf and ookinetes traversing the mosquito midgut epithelium at 27 hpbf, stained with $\alpha$-Pfc01 (green) and the female gamete/zygote/ookinete $\alpha$-Pfs25 (red) antibodies. Pre-immune (pre-imm) serum was used as negative control. DNA was stained with DAPI. Pre-immune serum was used as a negative control. BF, bright field; hpbf, hours post blood feeding. Scale bars, $5 \mu \mathrm{m}$. 
In $P$. falciparum, the $\alpha$-Pfc01 antibody detected a band of about $20 \mathrm{kDa}$ (predicted molecular weight of PfPIMMS01 is 19 $\mathrm{kDa}$ ) in $A$. coluzzii midguts infected $22 \mathrm{~h}$ earlier with $P$. falciparum NF54 (Figure 2D). This band was not detected in NF54 cultured schizonts or gametocytes (Figure S7). In indirect immunofluorescence assays (IFAs) using the $\alpha$-Pfc01 antibody, PfPIMMS01 was detected in gametes/early zygotes found in the blood bolus 2 hpbf, ookinetes in the mosquito blood bolus 19 hpbf, and ookinetes crossing the mosquito midgut epithelium (Figure 2E). Its localization varied from cytoplasmic in early developmental stages, mostly stages III and IV of the ookinete development, to totally peripheral in mature, midgutcrossing ookinetes.

\section{PIMMS57 Expression and Localization}

Using the non-functional c57::gfp parasite line and the $\alpha$-GFP antibody in western blot analysis, high levels of the PbPIMMS57::GFP fusion protein were detected in mature ookinetes, at the expected molecular weight of $117 \mathrm{kDa}$ (Figure 3A). This band was not detected in MBS or gametocytes or in the ANKA 2.34 background parasite line. Similarly, western blot analysis using the $c 57:: 3 x h a$ transgenic line and the $\alpha$-HA antibody confirmed expression of the PbPIMMS57::3xHA protein in the ookinete at the expected molecular weight and its absence from gametocytes (Figure 3B). The protein was mostly detected in the Triton insoluble fraction, indicating membrane association. No band was detected in the $c 507$ reference line.

To validate the ookinete expression of PbPIMMS57, the $\alpha$ Pbc57 peptide antibody was utilized. In westerns, the $\alpha$-Pbc57 antibody detected in ookinetes a band at about $90 \mathrm{kDa}$, close to the predicted molecular weight of PbPIMMS57. This band was not seen in MBS, gametocytes and the control $\Delta c 57$ (see below) knockout parasite line (Figure 3C). Fractionation assays revealed that, in ookinetes, PbPIMMS57 is mostly found in the Triton $\mathrm{X}-100$ insoluble fraction with low amounts also seen in the Triton X-100 soluble fraction (Figure 3D), suggesting that Pbc57 is membrane associated. These results agree with the prediction of transmembrane domains in this protein. The $\alpha$-Pbc57 peptide antibody did not work in IFAs.

IFAs on the developmentally compromised $c 57:: g f p$ and c57::3xha transgenic lines revealed cytoplasmic localization of the PbPIMMS57::GFP and PbPIMMS57::3xHA fusion proteins with bias for the ookinete convex side, especially for the former, pointing to a possible involvement of the protein with ookinete motility or invasion machinery (Figure 3E). However, given that both types of fusions lead to developmentally compromised parasites, these data must be interpreted with caution.

Similar analysis in $P$. falciparum using the $\alpha$-Pfc57 peptide antibody detected a clear band albeit of lower-than-expected molecular weight in western blots of midgut homogenates of $A$. coluzzii mosquitoes infected 22 h earlier with P. falciparum NF54 cultured gametocytes (Figure 3F). This in conjunction with additional low molecular weight bands could be attributed to degradation in the highly proteolytic environment of the midgut blood bolus. In IFAs, the $\alpha$-Pfc57 antibody showed that PfPIMMS57 is localized in the cytoplasm of gametes/early zygotes and intermediate ookinete developmental stages (mostly stages III and IV) obtained from the midgut blood bolus at 2 and 19 hpbf, respectively, with their distribution extending to the ookinete periphery (Figure 3G). Although it is difficult to decipher a clear membrane distribution of the signal, apparent similarities with the Pfs 25 signal in the ookinete periphery makes a membrane-associated localization of PfPIMMS57 highly probable.

\section{PIMMS22 Expression and Localization}

Western blot analysis using the $c 22:: g f p$ transgenic parasite line and $\alpha$-GFP antibody revealed high levels of the PbPIMMS22::GFP fusion protein in ookinete and oocyst derived sporozoites, at the expected molecular weight of $71 \mathrm{kDa}$ (Figure 4A). This band was not detected in the ANKA 2.34 background parasite line. IFAs on the $c 22:: g f p$ parasites allowed us to analyze the sub-cellular localization of the fusion protein. In Triton X-100 permeabilized blood stage gametocytes, PbPIMMS22::GFP was found to be localized on the cell periphery with the majority of the protein localizing in the cytoplasm (Figure 4B). Non-Triton X-100 permeabilized blood stage gametocytes are visibly less fluorescent than their Triton X-100 permeabilized counterparts. In Triton X100 permeabilized in vitro ookinetes, PbPIMMS22::GFP was clearly observed on the ookinete periphery, a result that is inconsistent with the predicted absence of a signal peptide or transmembrane domain (Figure 4C). Additional staining on non-Triton X-100 permeabilized ookinetes show some peripheral signal albeit much weaker than its Triton X-100 permeabilized counterpart suggesting that PbPIMMS22::GFP is mainly localized on the inner surface of the ookinete and not on the plasma membrane. IFAs on in vivo midgut epithelium invading ookinetes at $26 \mathrm{hpbf}$ also show surface localization of PbPIMMS22::GFP (Figure 4D). In midgut sporozoites, PbPIMMS22::GFP is found on the surface of both Triton X-100 and non-Triton X-100 permeabilized sporozoites (Figure 4E).

\section{Generation and Phenotypic Analysis of P. berghei Mutant Parasites}

Genetically modified $c 507$ P. berghei lines, designated $\Delta c 01, \Delta c 57$, and $\Delta c 22$, were generated by replacing most of the coding regions of PbPIMMS01, 57, and 22 with a modified Toxoplasma gondii pyrimethamine resistance (TgDHFR) expression cassette, respectively (Figures S8A-C). Integration of the disruption cassette and generation of clonal lines was confirmed by PCR and pulse field gel electrophoresis (Figures S8D-E).

Male gametogenesis in all three knockout parasite lines was assessed by counting exflagellation centers and found to be comparable to that of the $c 507$ parental line (Figure 5A). The macrogamete (female gamete) to ookinete conversion rates for all knockout lines were also comparable to that of the c507 parental reference line (Figure 5B). These two datasets indicated that gametocyte-ookinete development is not affected in any of these knockout parasite lines.

Next, we assessed the ability of the knockout lines to complete further developmental steps in $A$. coluzzii mosquitoes that were fed on mice each infected with a knockout parasite line or the 

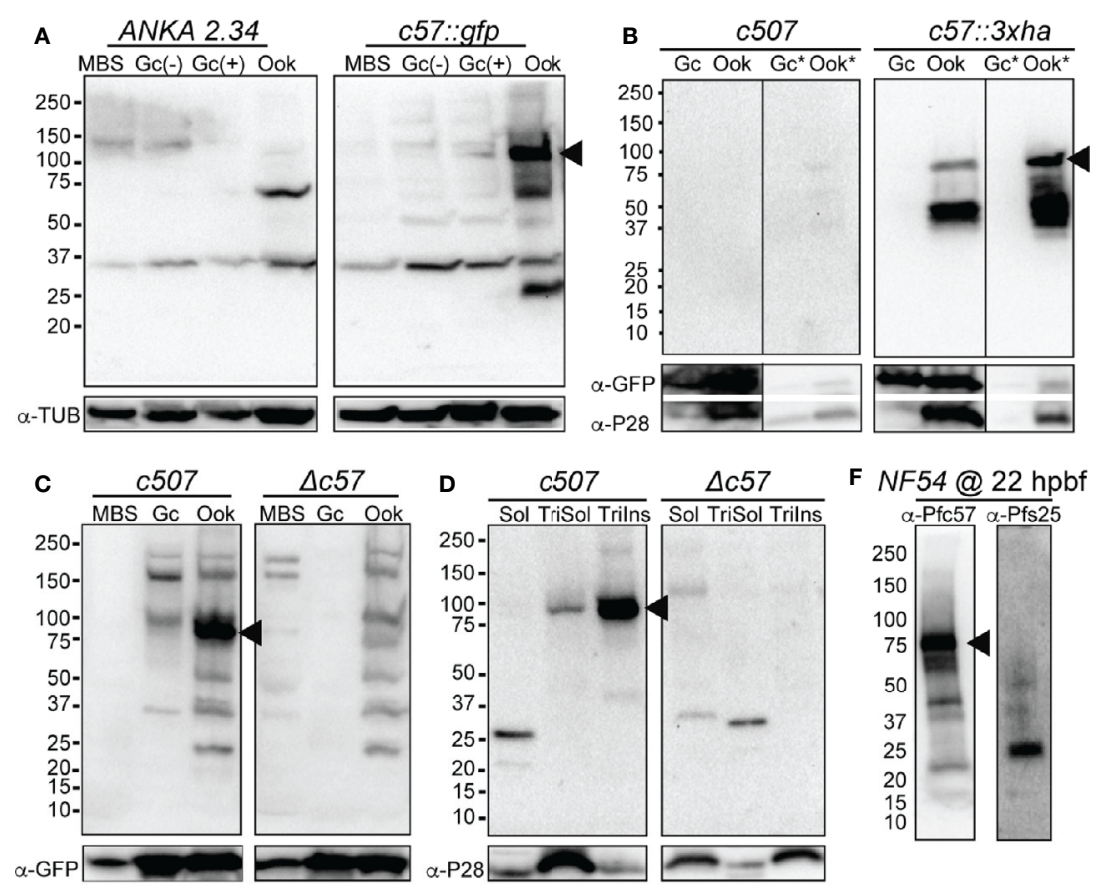

F NF54@ 22 hpbf

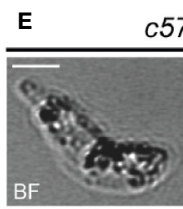

$57:: g f p$
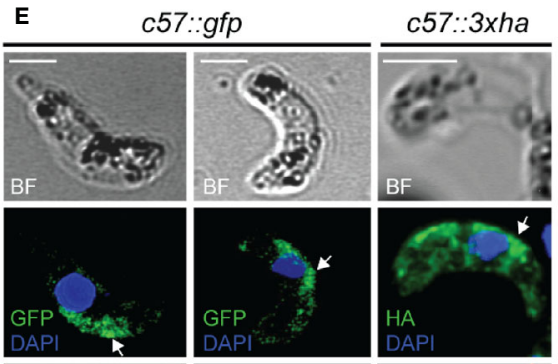

G
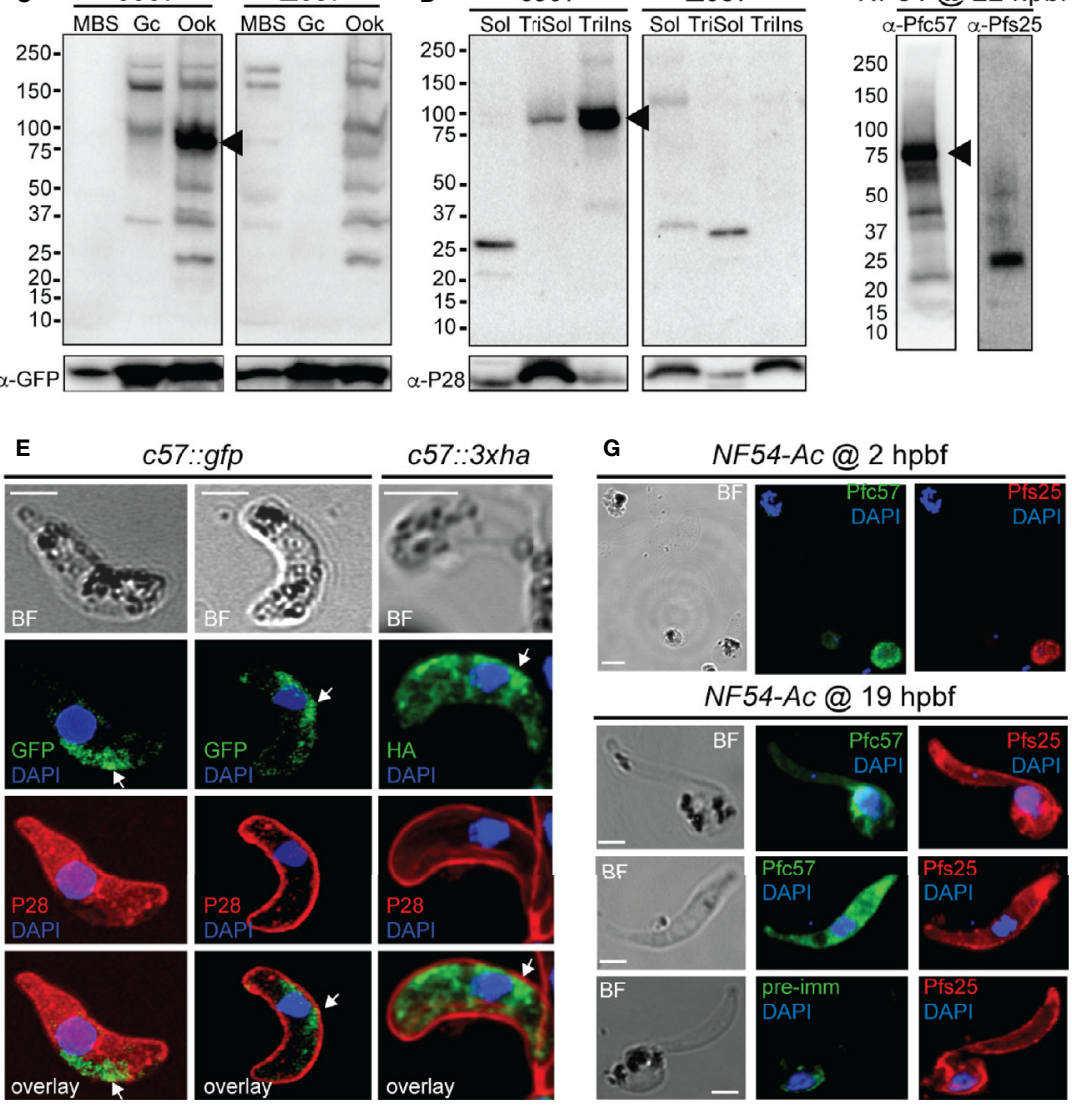

FIGURE 3 | PIMMS57 protein expression and localization. (A) Western blot analysis using $\alpha$-GFP antibody on whole cell lysates of the $P$. berghei c57::gfp parasites. The PbPIMMS57::GFP fusion protein band is indicated with a black arrowhead. The ANKA 2.34 parental parasite was used as a negative control. Tubulin was used a loading control. MBS, mixed blood stages; Gc(-), non-activated gametocytes; Gc(+), activated gametocytes; Ook, ookinetes. (B) Western blot analysis using the $\alpha-\mathrm{HA}$ antibody on Triton X-100 soluble lysates of in vitro gametocyte (Gc) and ookinetes (Ook) and Triton X-100 insoluble lysates (Gc* Ook ${ }^{\star}$ ) of c57::3xha. The c507 reference line (left panel) was used as a negative control. PbPIMMS57::HA fusion protein band is indicated with a black arrowhead. GFP and P28 were used as a loading and a stage specific control, respectively. (C) Western blot analysis using the $\alpha$-Pbc57 peptide antibody on whole cell lysates of MBS, Gc, and ookinetes of the $c 507$ parasite line. The PbPIMMS57 protein band is indicated with a black arrowhead. $\Delta c 57$ parasites were used as a negative control. GFP was used as a loading control. (D) Western blot analysis using the $\alpha$-Pbc57 antibody on fractionated in vitro ookinetes. The PbPIMMS57 protein band is indicated with a black arrowhead. $\Delta c 57$ ookinetes were used as a negative control. P28 was used as a loading control. Soluble (Sol), Triton X-100 soluble (TriSol) and Triton X-100 Insoluble (Trilnso) fractions are shown. (E) Immunofluorescence assays on ookinetes of PbPIMMS57 tagged with GFP and 3xHA stained with $\alpha$-GFP or $\alpha$-HA (green; white arrows) as well as with the ookinete surface $\alpha$-P28 (red). DNA was stained with DAPI. Staining of the c507 and ANKA 2.34 parental parasites were used as negative controls for the HA and GFP staining, respectively. Images are de-convoluted projections of confocal stacks. BF, bright field; Scale bars, $5 \mu$ m. (F) Western blot analysis using $\alpha$-Pfc57 antibody on whole cell lysates of $A$. coluzzi midguts at 22 hpbf. The PfPIMMS57 protein band is indicated with a black arrowhead. Pfs25 was used as a loading and stage specific control for $22 \mathrm{hpbf}$. (G) Immunofluorescence assays of $P$. falciparum NF54 parasites in mosquito blood bolus at 2 hpbf (top) and ookinetes in mosquito blood bolus at $19 \mathrm{hpbf}$ (bottom), stained with $\alpha$-Pfc57 (green) and the female gamete/zygote/ookinete $\alpha$-Pfs25 (red) antibodies. Preimmune serum was used as a negative control. DNA was stained with DAPI. Staining with pre-immune serum was used as a negative control. BF, bright field; Scale bars, $5 \mu \mathrm{m}$. 

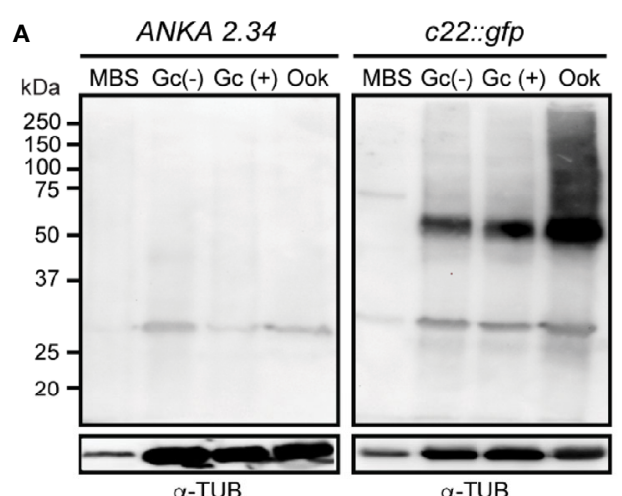

a-TUB
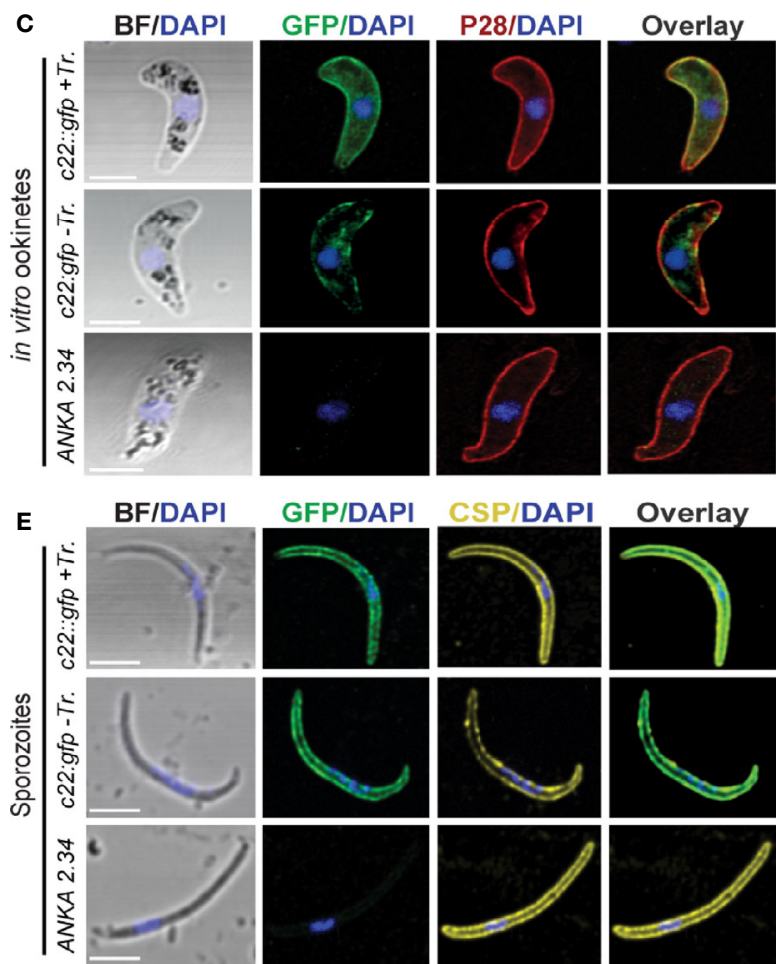
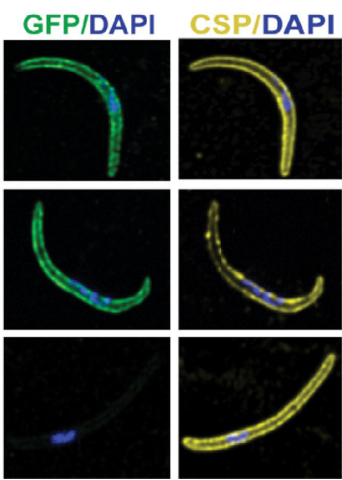

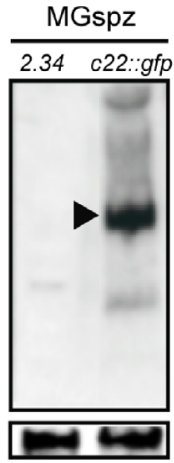

$\alpha-\operatorname{CSP}$

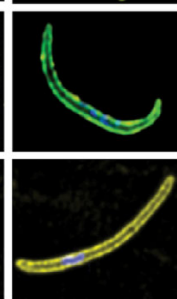

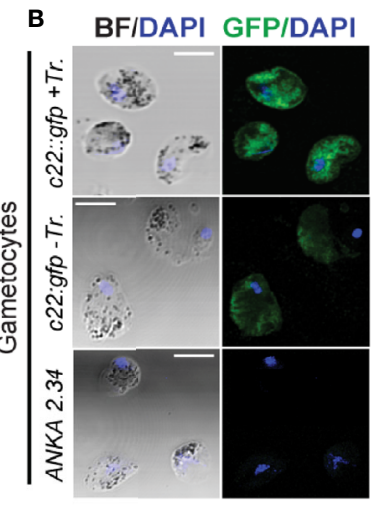

D midgut-invading ookinetes
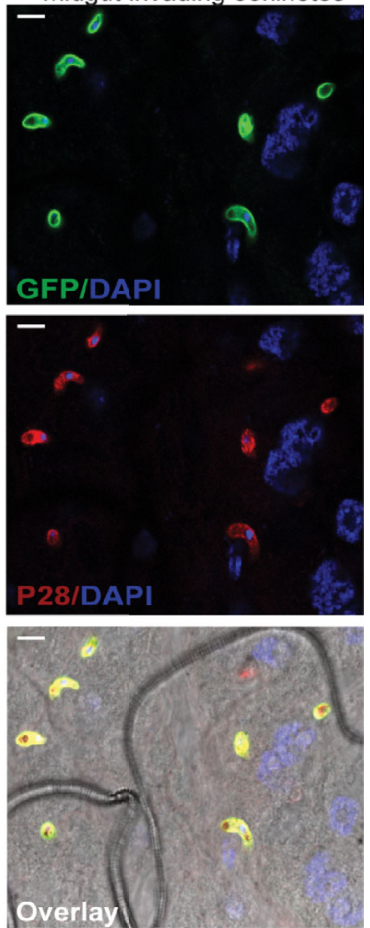

FIGURE 4 | PIMMS22 protein expression and localization. (A) Western blot analysis using $\alpha$-GFP antibody on whole cell lysates of $P$. berghei c22::gfp parasites. The PbPIMMS22::GFP fusion protein band is indicated with a black arrowhead. The ANKA 2.34 parental parasite line was used as a negative control. Tubulin was used a loading control in all parasite stages except from midgut sporozoites where CSP was used. MBS, mixed blood stages; Gc(-), non-activated gametocytes; Gc(+), activated gametocytes; Ook, ookinetes; MgSpz, midgut sporozoites. Immunofluorescence assays of c22::gfp blood stage gametocytes treated or not treated with Triton X-100 (Tr.) (B), in vitro ookinetes (C), ookinetes traversing the mosquito midgut epithelium at $26 \mathrm{hpbf}$ (D) and midgut sporozoites at $15 \mathrm{dpbf}$ (E), stained with $\alpha$-GFP (green), ookinete surface $\alpha$-P28 (red), or sporozoite surface $\alpha$-PbCSP (yellow) antibodies. DNA was stained with DAPI. Staining of the $2.34 \mathrm{wt}$ parental parasite was used as a negative control. Images are de-convoluted projections of confocal stacks. BF, bright field; Scale bars in (A-C, E) $5 \mu \mathrm{m}$; Scale bars in (D) $10 \mu \mathrm{m}$.

c507 parental line. Significant decreases in the numbers of oocysts present in the mosquito midguts at $8 \mathrm{dpbf}$ were observed for all the three knockout lines compared to c507 (Figure 5C, Table S3), indicating that ookinete to oocyst development is defective all three lines.

The ability of knockout mutant parasites to produce sporozoites was assessed by counting midgut and salivary gland sporozoites 15 and $21 \mathrm{dpbf}$, respectively. Compared to the $c 507$ reference line, significant decreases in midgut and salivary gland sporozoite numbers were observed for all three knockout lines (Figure 5D, Table S4). None of the three knockout parasites could be transmitted back to mice through mosquito bites carried out $21 \mathrm{dpbf}$, in all cases leading to termination in malaria transmission (Table S4).

Parasites displaying normal ookinete development but showing a defect during the ookinete to oocyst developmental 

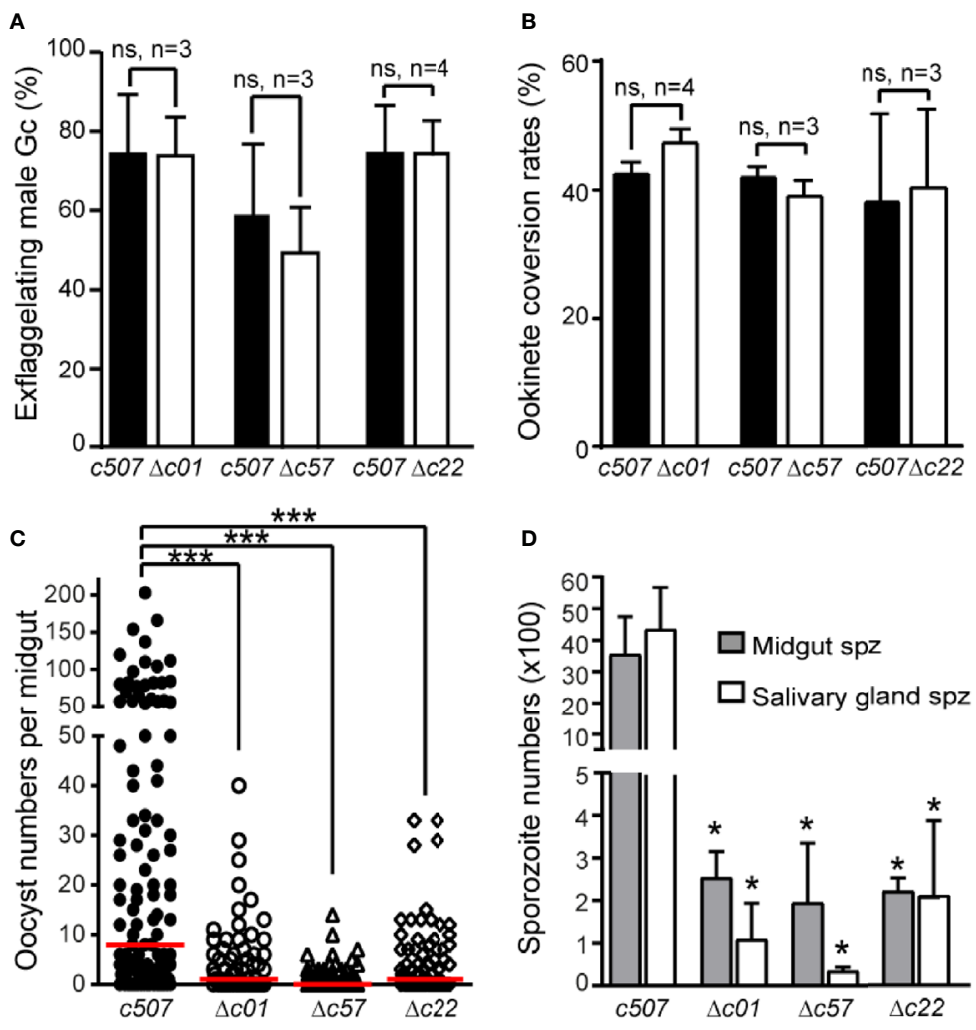

D

E
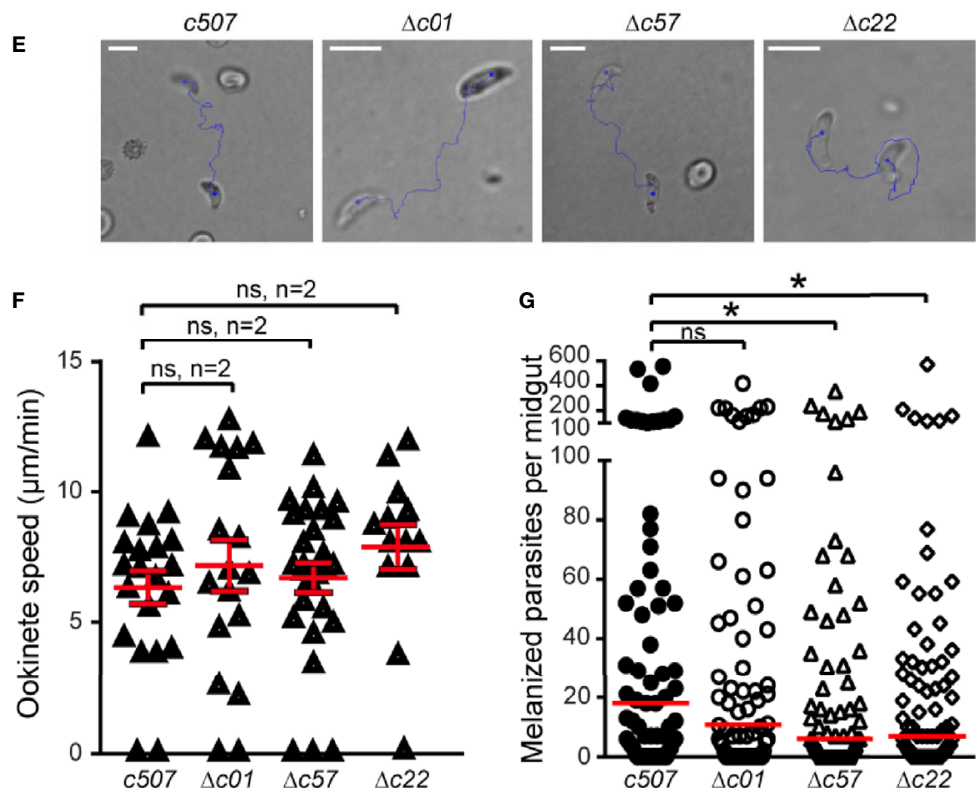

FIGURE 5 | Phenotypic analysis of $P$. berghei $\Delta \mathrm{c} 01, \Delta \mathrm{c} 57$, and $\Delta \mathrm{c} 22$ knockout mutants. Male gametocyte activation measured as percentage of exflagellating male gametocytes (A) and percentage of female gamete conversion to ookinetes (B) of the c507 reference and knockout parasites. ns, not significant; $\mathrm{n}$, number of biological replicates. Error bars indicate SEM. (C) $\Delta c 01, \Delta c 57$, and $\Delta c 22$ oocyst development at 8 dpbf in $A$. coluzzii. Red horizontal lines indicate median. ${ }^{\star \star \star} \mathrm{P}<0.0001$ using Mann-Whitney test. (D) $\Delta c 01, \Delta c 57$, and $\Delta c 22$ midgut (MgSpz) and salivary gland sporozoite (SgSpz) numbers at 15 and $21 \mathrm{dpbf}$ respectively in A. coluzzii. (E) Representative instances of $c 507$ reference and $\Delta c 01, \Delta c 57$, and $\Delta c 22$ knockout ookinetes from gliding motility assays. Blue lines show ookinete gliding traces captured for $2 \mathrm{~min}$. Note the gliding helical motility that is characteristic of wt ookinete observed in these ookinetes. Scale bars, $10 \mu \mathrm{m}$. (F) Speed of c507 reference and knockout ookinetes measured from time-lapse microscopy. ns, not significant; n, number of biological replicates. Red horizontal lines indicate mean and error bars show SEM. (G) Melanized ookinete numbers in CTL4 kd A. coluzzii infected with c507 reference and knockout parasite lines. Red lines indicate median; ns, not significant; *P $<0.05$ using Mann-Whitney test. 
transition could be a result of a defect in ookinete motility and/or a midgut invasion and traversal. To investigate for motility defects, speed measurements of ookinetes were carried out. $\Delta c 01, \Delta c 57$, and $\Delta c 22$ in vitro cultured ookinetes exhibited movements with speed not significantly different to that of the c507 wt ookinete (Figures 5E, F and Movies S1-8).

Next, we investigated for invasion defects by carrying out mosquito infections in A. coluzzii mosquitoes silenced for C-type lectin CTL4 using RNA interference. Knockdown of CTL4 leads to melanization of ookinetes immediately after they have traversed the midgut epithelium and been exposed to the haemocoel in the basal sub-epithelial space thereby providing a means to visualize and enumerate ookinetes that successfully traverse the midgut epithelium. The number of melanized $\Delta c 01$ parasites was not significantly different to the $c 507$ reference parasite line $(\mathrm{p}=0.3971)$ (Figure 5G, Table S5), suggesting that $\Delta c 01$ ookinetes can readily invade and traverse the midgut epithelium but are defective at the ookinete-to-oocyst transition stage resulting in the significant decrease in the oocyst numbers observed. However, compared to the c507 parasite, significantly lower numbers of melanized $\Delta c 57$ $(\mathrm{p}=0.0337)$ and $\Delta c 22(\mathrm{p}=0.0487)$ ookinetes was observed, indicating that $\Delta c 57$ and $\Delta c 22$ ookinetes are defective in midgut invasion. However, the documented reduction in midgut invasion capacity of $\Delta c 57$ and $\Delta c 22$ ookinetes cannot fully explain the massive reduction in oocyst numbers, suggesting that like $\Delta c 01, \Delta c 57$, and $\Delta c 22$ are also defective for ookinete to oocyst transition.

The ookinete to oocyst developmental transition potential of $\Delta c 01, \Delta c 57$, and $\Delta c 22$ ookinetes was further assessed by bypassing the midgut epithelium entirely and injecting these ookinetes into the haemocoel of $A$. coluzzii mosquitoes. By skipping midgut epithelium invasion, $\Delta c 57$ ookinetes can transform to oocysts that produce salivary gland sporozoites at numbers comparable to those of the $c 507$ reference line (Table 1). These sporozoites could also be transmitted to mice in transmission experiments. In this experiment, while the mean $\Delta c 01$ salivary gland sporozoite number was significantly lower than the $c 507$ parasite, high $\Delta c 01$ salivary gland sporozoite numbers observed in some replicates resulted in transmission to mice while lower salivary gland sporozoite numbers resulted in no transmission suggesting that $\Delta c 01$ transmission efficiency is dependent on sporozoite numbers. For the $\Delta c 22$ parasite line, the significantly smaller number of salivary gland sporozoites produced were still not able to initiate transmission (Table 1).

\section{DISCUSSION}

To establish a successful infection in the mosquito, Plasmodium parasites must within $24 \mathrm{~h}$ of uptake into the mosquito midgut, fertilize to form ookinetes that have to invade and traverse the midgut epithelium and form oocysts on the basal side of the epithelium. The molecular processes driving the Plasmodium ookinete to oocyst developmental transition remain relatively under characterized. In this study, we have identified PIMMS01, PIMMS57, and PIMMS22 as being important factors for ookinete to oocyst transition and essential for malaria transmission. While gametocyte to ookinete development is not affected in the mutant knockout parasites, they all display severe defects in oocyst formation. The midgut invasion capabilities of these knockout parasites suggest that other factors come into play to block this ookinete to oocyst transitional step. The decrease in number of subsequent sporozoites eventually result in an abolishment of malaria transmission. The observed reduction in sporozoites may not be merely an effect of the oocyst defective phenotype. Since the gene knock out system used in this study is not regulatable, gene functions cannot be assessed past the point of their initial essential action. This means that additional functions of these genes past the ookinete to oocyst developmental transition in oocyst and sporozoite development and in malaria transmission cannot be ruled out. This is especially true for PIMMS22 which shows peak transcript expression during oocyst sporozoite development and its concomitant protein is highly expressed also at this stage.

Amongst genes reported to function during the ookinete to oocyst developmental transition, an overwhelming defect before and at entry into the midgut is observed. The ookinete to oocyst defective mutants of CTRP, PPKL, CDPK3, GC $\beta, D H H C 3, O M D$, and the alveolins IMC1 $b$ and $h$ are defective in ookinete motility (Dessens et al., 1999; Siden-Kiamos et al., 2006; Tremp et al., 2008;

TABLE 1 | Sporozoite development and infectivity upon ookinete injection in the haemocoel.

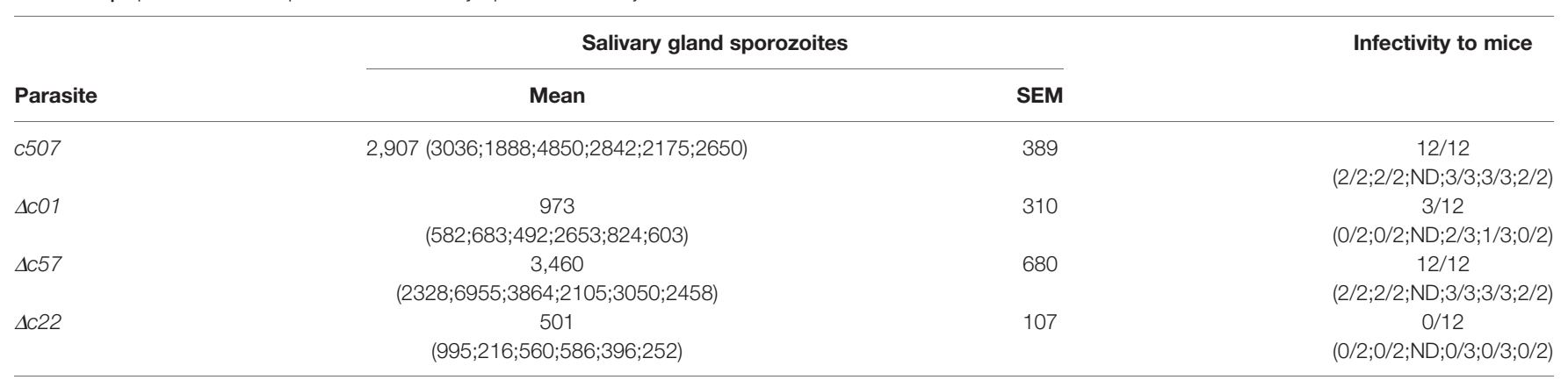

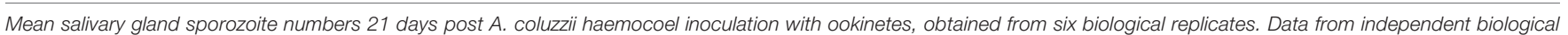

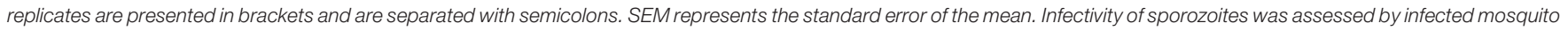

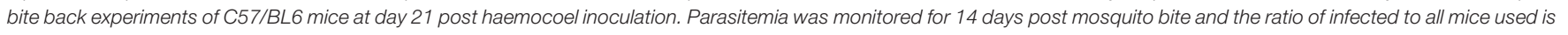
shown. ND, not determined. 
Moon et al., 2009; Guttery et al., 2012; Volkmann et al., 2012; Hopp et al., 2016; Currà et al., 2019), CHT mutants are defective in ookinete penetration of the peritrophic matrix (Dessens et al., 2001) and P25, P28, SOAP, PPLP3, PPLP4, PPLP5, PSOP2, 7, and 9 and Enolase mutants are defective in ookinete midgut invasion (Tomas et al., 2001; Dessens et al., 2003; Kadota et al., 2004; Ecker et al., 2007; Ecker et al., 2008; Ghosh et al., 2011; Deligianni et al., 2018). Only null mutants of CelTOS, MISFIT, PPM5, AP2-O4, PIMMS2, and PIMMS43 have been identified to have a midgut invasion independent phenotype with their null mutant ookinetes able to invade the midgut epithelium but have problems in establishing an oocyst infection (Kariu et al., 2006; Bushell et al., 2009; Guttery et al., 2014; Modrzynska et al., 2017; Ukegbu et al., 2017; Ukegbu et al., 2020). Cell traversal upon midgut invasion is not compromised in $\Delta c 01$ mutant ookinetes like that observed in the oocyst formation defective PIMMS2 and CelTOS mutants (Kariu et al., 2006; Ukegbu et al., 2017), as melanized $\Delta c 01$ mutant ookinetes must have traverse the midgut epithelium to reach the basal lamina where the melanization response occurs (Osta et al., 2004). In the MISFIT and PPM5 mutants, the reduced number of oocysts formed are smaller and fail to complete sporulation (Bushell et al., 2009; Guttery et al., 2014). Our observations show that the small number of oocysts formed by the $\Delta c 01, \Delta c 57$, and $\Delta c 22$ parasites are morphologically normal with sizes comparable to $w t$ oocysts suggesting these proteins function differently from MISFIT and PPM5.

The observation that parasite development is rescued when $\Delta c 57$ ookinetes bypass the epithelium, suggests that the viability of these ookinetes and their inherent ability to differentiate into the oocyst is not affected. This implies that the ookinete to oocyst developmental transition is only impaired when ookinetes must cross the midgut wall. This type of phenotype has been previously observed in the SHLP1 mutant (Patzewitz et al., 2013). There, it has been suggested that developmental defects in the ookinete such as in microneme deficiency, results in a block in oocyst formation following midgut invasion which is restored if mutant ookinetes are injected into the haemocoel (Patzewitz et al., 2013). However, this may not be the case for $\Delta p b c 57$, as ookinete microneme deficiency has been previously shown not to be essential for the ookinete transformation to oocyst (Bushell et al., 2009). Whether any ookinete morphological/developmental defects are involved with the seemingly dual phenotype of the $\Delta p b c 57$ parasite requires further investigation. Indeed, the ookinete injection experiment has shown that the reduced $\Delta c 57$ oocyst and salivary gland sporozoites obtained with the natural route of infection is a direct result of the reduced number of oocysts and not due to any additional function of PIMMS57 at the oocyst and sporozoite developmental stages.

The ookinete injection phenotypes of $\Delta c 01$ and $\Delta c 22$ indicate that the defective oocyst formation phenotypes are independent of midgut invasion suggesting that the viability of these ookinetes are overall affected in vivo in the mosquito. While the sporozoite numbers for $\Delta c 01$ and $\Delta c 22$ appeared to be higher than those obtained from the direct blood feeding, the observation that the sporozoite numbers produced, following injection, are on average lower than the $w t$ parasite indicates that their development is still impaired. As it is impossible to accurately assess ookinete to oocyst transformation in this experiment (oocyst enumeration upon ookinete haemocoel injection is unreliable as oocysts are formed everywhere in the haemocoelbathed tissues) (Paskewitz and Shi, 2005), it is possible that $\Delta c 01$ and $\Delta c 22$ ookinetes can transform to oocysts but the reduced sporozoite numbers are the result of impaired oocyst sporogony or defect at the transition of oocyst sporozoites to the salivary glands. As expected and has been previously observed (Churcher et al., 2017), $\Delta c 01$ salivary gland sporozoite numbers following ookinete injection significantly affects transmission. Finally, the observation that $\Delta c 22$ sporozoites produced following ookinete injection could still not be transmitted suggests putative additional functions of PIMMS22 during this stage.

The observed impaired parasite development suggests putative roles of PIMMS01, PIMMS57, and PIMMS22 proteins in interaction with the midgut to either promote ookinete fitness or ookinete-to-oocyst differentiation. PIMMS01 and PIMMS57 are predicted to be secreted and membrane-bound, respectively, while PIMMS22 does not show any putative transmembrane domains but is clearly localized at the ookinete periphery, possibly the inner membrane complex. While the results showing that PIMMS01 and PIMMS57 are also localized at the ookinete periphery must be interpreted with caution, midgut crossing ookinetes show a clear peripheral localization of PIMMS01 and PIMMS57 has been previously also shown by others to localize on the ookinete surface (Zheng et al., 2016). All these are suggestive of a capability of these proteins to interact, directly or indirectly, with the midgut environment.

The mosquito midgut epithelium via effectors of the JNK pathway has been proposed to actively mark invading ookinetes for killing by the mosquito complement-like system (de Almeida Oliveira et al., 2012). The GPI-anchored ookinete surface protein Pfs47 has been suggested to protect $P$. falciparum ookinetes against attack by this system (Molina-Cruz et al., 2013), and we have previously shown that the ookinete surface protein PIMMS43 is also essential in protecting ookinetes by complement-like responses (Ukegbu et al., 2020). While PIMMS01, PIMMS57, and PIMMS22 may directly interact with the midgut, much like Pfs47 whose receptor in the midgut epithelium has been recently identified (Molina-Cruz et al., 2020) to promote ookinete survival, an alternative explanation is that loss of function of these proteins may bear a fitness cost on ookinetes. In the midgut, where oxidative stress is high due to the blood meal (Turturice et al., 2013) and $P$. berghei infection of the midgut has been shown to exacerbate the production of reactive oxygen species (ROS) (Molina-Cruz et al., 2008), ookinetes lacking such proteins are expected to be compromised.

A clue to the possible functions of these proteins is the presence of defining signatures in their amino acid sequences. Apart from the signal peptide, PIMMS01 is devoid of any other domains that could predict its putative function. The identification of putative PP1c binding motifs in P. falciparum PIMMS57 suggests that it may function through PP1. In eukaryotes, PP1 is essential for cell cycle progression (Bollen et al., 2009). The exact role of PP1 in Plasmodium has not been 
deciphered yet due to its essentiality in asexual blood stages (Guttery et al., 2014). Nevertheless, the identification of several PP1c interacting proteins that can modulate the activity of this enzyme (Daher et al., 2006; Fréville et al., 2012; Hollin et al., 2016), including the gametocyte exported protein GEXP15 that is important for both blood stage development and oocyst formation (Hollin et al., 2019), could suggest additional and important roles of PP1-like activity during sexual development in the mosquito. The putative physical interaction between PfPIMMS57 and PfPP1c remains to be confirmed in functional interaction studies. The lack of PP1c binding motifs in the rest of the PIMMS57 orthologs suggest that these may function independently of PP1.

Like PIMMS01, PIMMS22 lacks any domain that could predict its putative function. Its localization and putative interacting partners could however point to the function of this protein. While we have shown PbPIMMS22 to localize at the ookinete periphery and putatively on the inner surface of the ookinete, how it achieves this without a predicted signal peptide or transmembrane domain remains to be investigated. A hypothesis is that PIMMSS22 could be interacting with other proteins located on the inner surface of the ookinete. This theory is supported by the observation that in P. yoelii sporozoites, PIMMS22 is found in a complex with several alveolin proteins of the subpellicular network and glideosome-associated proteins of the inner membrane complex (Muñoz et al., 2017). This interaction with the alveolin proteins could also be extended to the ookinete as some members of the SPN (subpellicular network) and IMC (inner membrane complex) are conserved and utilized across the ookinete and sporozoite stages (Morrissette and Sibley, 2002; Santos et al., 2009; Al-Khattaf et al., 2015). Proteins associated with the SPN and IMC are mostly linked to functions relating to cell motility (Tremp and Dessens, 2011; Volkmann et al., 2012; Frénal et al., 2017); however, this is not the case for PIMMS22 as no defect in ookinete motility is observed. Any putative functional interactions between PIMMS22 and proteins associated with the SPN and IMC will have to be further investigated in colocalization and pull-down experiments.

Despite the unknown exact molecular mechanisms that PIMMS01, PIMMS57, and PIMMS22 utilize to promote the ookinete to oocyst developmental transition, these proteins are good targets for the development of transmission blocking interventions. Two approaches are envisaged. First, and like the current frontline transmission blocking vaccine candidates Pfs230, Pfs48/45, and Pfs25 that target gametocyte/ookinete surface proteins, antibodies against these proteins can be generated in the human serum which, when ingested by mosquitoes together with gametocytes, interfere with the function of these proteins and block transmission to a new host (Nikolaeva et al., 2015). While this approach has been hampered by the difficulty in recombinant expression of full length and correctly folded Plasmodium proteins in a high throughput manner (Nikolaeva et al., 2020), the identification of small proteins that can be easily expressed such as PIMMS01 bears hopes for this approach. An alternative approach includes the creation of genetically modified mosquitoes which express single-chain antibodies that bind these proteins conferring refractoriness to infection and eventual blocking of malaria transmission (Isaacs et al., 2011; Gantz et al., 2015). These transgenes can be spread within wild mosquito populations through gene drive mechanisms (e.g. CRISPR/Cas9) leading to sustainable local malaria elimination (Carballar-Lejarazú and James, 2017).

\section{DATA AVAILABILITY STATEMENT}

The original contributions presented in the study are included in the article/Supplementary Material. Further inquiries can be directed to the corresponding author.

\section{ETHICS STATEMENT}

The animal study was reviewed and approved by Animal Welfare and Ethical Review Body (AWERB), Imperial College London.

\section{AUTHOR CONTRIBUTIONS}

Conceptualization, DV. Methodology, CU and DV. Formal analysis, CU and DV. Investigation, CU and DV. Resources, GC and DV. Data curation, CU and DV. Writing paper, CU, GC, and DV. Supervision, DV. Project administration, GC and DV. Funding acquisition, GC and DV. All authors contributed to the article and approved the submitted version.

\section{FUNDING}

The work was funded by a Wellcome Trust Investigator Award $(107983 / Z / 15 / Z)$ to GC, a Wellcome Trust Project grant $(093587 / \mathrm{Z} / 10 / \mathrm{Z})$ to GC and DV, and a Bill and Melinda Gates Foundation grant (OPP1158151) to GC.

\section{ACKNOWLEDGMENTS}

The authors thank Temesgen M. Kebede for assistance with $P$. berghei culturing and mosquito rearing, Julia Cai for providing some P. falciparum NF54 RNA for qPCR assays, Maria Grazia Inghilterra for $P$. falciparum culturing and Rajeev Rai and Chrysanthi Taxiarchi for technical assistance with the ko constructs for the generation of the mutant parasites and for mosquito infections.

\section{SUPPLEMENTARY MATERIAL}

The Supplementary Material for this article can be found online at: https://www.frontiersin.org/articles/10.3389/fcimb.2021. 634273/full\#supplementary-material 


\section{REFERENCES}

Akinosoglou, K. A., Bushell, E. S., Ukegbu, C. V., Schlegelmilch, T., Cho, J. S., Redmond, S., et al. (2015). Characterization of p lasmodium developmental transcriptomes in a nopheles gambiae midgut reveals novel regulators of malaria transmission. Cell. Microbiol. 17 (2), 254-268. doi: 10.1111/cmi.12363

Al-Khattaf, F. S., Tremp, A. Z., and Dessens, J. T. (2015). Plasmodium alveolins possess distinct but structurally and functionally related multi-repeat domains. Parasitol. Res. 114, 631-639. doi: 10.1007/s00436-014-4226-9

Armenteros, J. J. A., Tsirigos, K. D., Sønderby, C. K., Petersen, T. N., Winther, O., Brunak, S., et al. (2019). Signalp 5.0 improves signal peptide predictions using deep neural networks. Nat. Biotechnol. 37, 420-423. doi: 10.1038/s41587-0190036-z

Barr, PJ, Green, KM, Gibson, HL, Bathurst, IC, Quakyi, IA, and Kaslow, DC (1991). Recombinant Pfs25 protein of Plasmodium falciparum elicits malaria transmission-blocking immunity in experimental animals. J. Exp. Med. 174 (5), 1203-1208. doi: 10.1084/jem.174.5.1203

Beetsma, A., van de Wiel, T. J., Sauerwein, R., and Eling, W. (1998). Plasmodium bergheianka: Purification of large numbers of infectious gametocytes. Exp. Parasitol. 88, 69-72. doi: 10.1006/expr.1998.4203

Bollen, M., Gerlich, D. W., and Lesage, B. (2009). Mitotic phosphatases: From entry guards to exit guides. Trends Cell Biol. 19, 531-541. doi: 10.1016/ j.tcb.2009.06.005

Braks, J. A., Franke-Fayard, B., Kroeze, H., Janse, C. J., and Waters, A. P. (2006). Development and application of a positive-negative selectable marker system for use in reverse genetics in plasmodium. Nucleic Acids Res. 34, e39-e39. doi: 10.1093/nar/gnj033

Bushell, E. S., Ecker, A., Schlegelmilch, T., Goulding, D., Dougan, G., Sinden, R. E., et al. (2009). Paternal effect of the nuclear formin-like protein misfit on plasmodium development in the mosquito vector. PLoS Pathog. 5, e1000539. doi: 10.1371/journal.ppat.1000539

Carballar-Lejarazú, R., and James, A. A. (2017). Population modification of anopheline species to control malaria transmission. Pathog. Global Health 111, 424-435. doi: 10.1080/20477724.2018.1427192

Churcher, T. S., Sinden, R. E., Edwards, N. J., Poulton, I. D., Rampling, T. W., Brock, P. M., et al. (2017). Probability of transmission of malaria from mosquito to human is regulated by mosquito parasite density in naive and vaccinated hosts. PLoS Pathog. 13, e1006108. doi: 10.1371/ journal.ppat.1006108

Costa, G., Gildenhard, M., Eldering, M., Lindquist, R., Hauser, A., Sauerwein, R., et al. (2018). Non-competitive resource exploitation within mosquito shapes within-host malaria infectivity and virulence. Nat. Commun. 9, 1-11. doi: 10.1038/s41467-018-05893-z

Currà, C., Kehrer, J., Lemgruber, L., Silva, P. A., Bertuccini, L., Superti, F., et al. (2019). Malaria transmission through the mosquito requires the function of the omd protein. PLoS One 14, e0222226. doi: 10.1371/journal.pone.0222226

Daher, W., Browaeys, E., Pierrot, C., Jouin, H., Dive, D., Meurice, E., et al. (2006). Regulation of protein phosphatase type 1 and cell cycle progression by pflrr1, a novel leucine-rich repeat protein of the human malaria parasite plasmodium falciparum. Mol. Microbiol. 60, 578-590. doi: 10.1111/j.13652958.2006.05119.x

de Almeida Oliveira, G., Lieberman, J., and Barillas-Mury, C. (2012). Epithelial nitration by a peroxidase/nox 5 system mediates mosquito antiplasmodial immunity. Science 335, 856-859. doi: 10.1126/science.1209678

Deligianni, E., Silmon de Monerri, N. C., McMillan, P. J., Bertuccini, L., Superti, F., Manola, M., et al. (2018). Essential role of plasmodium perforin-like protein 4 in ookinete midgut passage. PLoS One 13, e0201651. doi: 10.1371/ journal.pone.0204083

Dessens, J. T., Beetsma, A. L., Dimopoulos, G., Wengelnik, K., Crisanti, A., Kafatos, F. C., et al. (1999). Ctrp is essential for mosquito infection by malaria ookinetes. EMBO J. 18, 6221-6227. doi: 10.1093/emboj/18.22.6221

Dessens, J. T., Mendoza, J., Claudianos, C., Vinetz, J. M., Khater, E., Hassard, S., et al. (2001). Knockout of the rodent malaria parasite chitinase pbcht1 reduces infectivity to mosquitoes. Infect. Immun. 69, 4041-4047. doi: 10.1128/ IAI.69.6.4041-4047.2001

Dessens, J. T., Sidén-Kiamos, I., Mendoza, J., Mahairaki, V., Khater, E., Vlachou, D., et al. (2003). Soap, a novel malaria ookinete protein involved in mosquito midgut invasion and oocyst development. Mol. Microbiol. 49, 319-329. doi: 10.1046/ j.1365-2958.2003.03566.x

Dong, Y., Aguilar, R., Xi, Z., Warr, E., Mongin, E., and Dimopoulos, G. (2006). Anopheles gambiae immune responses to human and rodent plasmodium parasite species. PLoS Pathog. 2, e52. doi: 10.1371/journal.ppat.0020052

Ecker, A., Pinto, S. B., Baker, K. W., Kafatos, F. C., and Sinden, R. E. (2007). Plasmodium berghei: Plasmodium perforin-like protein 5 is required for mosquito midgut invasion in anopheles stephensi. Exp. Parasitol. 116, 504508. doi: 10.1016/j.exppara.2007.01.015

Ecker, A., Bushell, E. S., Tewari, R., and Sinden, R. E. (2008). Reverse genetics screen identifies six proteins important for malaria development in the mosquito. Mol. Microbiol. 70, 209-220. doi: 10.1111/j.1365-2958.2008.06407.x

Frénal, K., Dubremetz, J.-F., Lebrun, M., and Soldati-Favre, D. (2017). Gliding motility powers invasion and egress in apicomplexa. Nat. Rev. Microbiol. 15, 645. doi: $10.1038 /$ nrmicro.2017.86

Fréville, A., Landrieu, I., García-Gimeno, M. A., Vicogne, J., Montbarbon, M., Bertin, B., et al. (2012). Plasmodium falciparum inhibitor-3 homolog increases protein phosphatase type 1 activity and is essential for parasitic survival. J. Biol. Chem. 287, 1306-1321. doi: 10.1074/jbc.M111.276865

Gantz, V. M., Jasinskiene, N., Tatarenkova, O., Fazekas, A., Macias, V. M., Bier, E., et al. (2015). Highly efficient cas9-mediated gene drive for population modification of the malaria vector mosquito anopheles stephensi. Proc. Natl. Acad. Sci. 112, E6736-E6743. doi: 10.1073/pnas.1521077112

Ghosh, A. K., Coppens, I., Gårdsvoll, H., Ploug, M., and Jacobs-Lorena, M. (2011). Plasmodium ookinetes coopt mammalian plasminogen to invade the mosquito midgut. Proc. Natl. Acad. Sci. 108, 17153-17158. doi: 10.1073/ pnas. 1103657108

Guttery, D. S., Poulin, B., Ferguson, D. J., Szöőr, B., Wickstead, B., Carroll, P. L., et al. (2012). A unique p rotein p hosphatase with k elch-l ike domains (ppkl) in plasmodium modulates ookinete differentiation, motility and invasion. PLoS Pathog. 8, e1002948. doi: 10.1371/journal.ppat.1002948

Guttery, D. S., Poulin, B., Ramaprasad, A., Wall, R. J., Ferguson, D. J., Brady, D., et al. (2014). Genome-wide functional analysis of plasmodium protein phosphatases reveals key regulators of parasite development and differentiation. Cell Host Microbe 16, 128-140. doi: 10.1016/ j.chom.2014.05.020

Habtewold, T., Povelones, M., Blagborough, A. M., and Christophides, G. K. (2008). Transmission blocking immunity in the malaria non-vector mosquito anopheles quadriannulatus species a. PLoS Pathog. 4, e1000070. doi: 10.1371/ journal.ppat.1000070

Habtewold, T., Tapanelli, S., Masters, E. K., Hoermann, A., Windbichler, N., and Christophides, G. K. (2019). Streamlined smfa and mosquito dark-feeding regime significantly improve malaria transmission-blocking assay robustness and sensitivity. Malaria J. 18, 1-11. doi: 10.1186/s12936-019-2663-8

Hall, N., Karras, M., Raine, J. D., Carlton, J. M., Kooij, T. W., Berriman, M., et al. (2005). A comprehensive survey of the plasmodium life cycle by genomic, transcriptomic, and proteomic analyses. Science 307, 82-86. doi: 10.1126/ science. 1103717

Hollin, T., De Witte, C., Lenne, A., Pierrot, C., and Khalife, J. (2016). Analysis of the interactome of the ser/thr protein phosphatase type 1 in plasmodium falciparum. BMC Genomics 17, 246. doi: 10.1186/s12864-016-2571-z

Hollin, T., De Witte, C., Fréville, A., Guerrera, I. C., Chhuon, C., Saliou, J.-M., et al. (2019). Essential role of gexp15, a specific protein phosphatase type 1 partner, in plasmodium berghei in asexual erythrocytic proliferation and transmission. PLoS Pathog. 15, e1007973. doi: 10.1371/journal.ppat.1007973

Hopp, C. S., Balaban, A. E., Bushell, E. S., Billker, O., Rayner, J. C., and Sinnis, P. (2016). Palmitoyl transferases have critical roles in the development of mosquito and liver stages of plasmodium. Cell. Microbiol. 18, 1625-1641. doi: $10.1111 / \mathrm{cmi} .12601$

Howick, V. M., Russell, A. J., Andrews, T., Heaton, H., Reid, A. J., Natarajan, K., et al. (2019). The malaria cell atlas: Single parasite transcriptomes across the complete plasmodium life cycle. Science 365, eaaw2619. doi: 10.1126/ science.aaw2619

Isaacs, A. T., Li, F., Jasinskiene, N., Chen, X., Nirmala, X., Marinotti, O., et al. (2011). Engineered resistance to plasmodium falciparum development in transgenic anopheles stephensi. PLoS Pathog. 7, e1002017. doi: 10.1371/ journal.ppat.1002017 
Janse, C., and Waters, A. (1995). Plasmodium berghei: The application of cultivation and purification techniques to molecular studies of malaria parasites. Parasitol. Today 11, 138-143. doi: 10.1016/0169-4758(95)80133-2

Janse, C. J., Franke-Fayard, B., Mair, G. R., Ramesar, J., Thiel, C., Engelmann, S., et al. (2006a). High efficiency transfection of plasmodium berghei facilitates novel selection procedures. Mol. Biochem. Parasitol. 145, 60-70. doi: 10.1016/ j.molbiopara.2005.09.007

Janse, C. J., Ramesar, J., and Waters, A. P. (2006b). High-efficiency transfection and drug selection of genetically transformed blood stages of the rodent malaria parasite plasmodium berghei. Nat. Protoc. 1, 346. doi: 10.1038/ nprot.2006.53

Kadota, K., Ishino, T., Matsuyama, T., Chinzei, Y., and Yuda, M. (2004). Essential role of membrane-attack protein in malarial transmission to mosquito host. Proc. Natl. Acad. Sci. 101, 16310-16315. doi: 10.1073/pnas.0406187101

Kaiser, K., Matuschewski, K., Camargo, N., Ross, J., and Kappe, S. H. (2004). Differential transcriptome profiling identifies plasmodium genes encoding preerythrocytic stage-specific proteins. Mol. Microbiol. 51, 1221-1232. doi: 10.1046/j.1365-2958.2003.03909.x

Käll, L., Krogh, A., and Sonnhammer, E. L. (2007). Advantages of combined transmembrane topology and signal peptide prediction-the phobius web server. Nucleic Acids Res. 35, W429-W432. doi: 10.1093/nar/gkm256

Kariu, T., Ishino, T., Yano, K., Chinzei, Y., and Yuda, M. (2006). Celtos, a novel malarial protein that mediates transmission to mosquito and vertebrate hosts. Mol. Microbiol. 59, 1369-1379. doi: 10.1111/j.1365-2958.2005.05024.x

Lasonder, E., Rijpma, S. R., van Schaijk, B. C., Hoeijmakers, W. A., Kensche, P. R., Gresnigt, M. S., et al. (2016). Integrated transcriptomic and proteomic analyses of p. Falciparum gametocytes: Molecular insight into sex-specific processes and translational repression. Nucleic Acids Res. 44, 6087-6101. doi: 10.1093/nar/ gkw536

Le Roch, K. G., Zhou, Y., Blair, P. L., Grainger, M., Moch, J. K., Haynes, J. D., et al. (2003). Discovery of gene function by expression profiling of the malaria parasite life cycle. Science 301, 1503-1508. doi: 10.1126/science.1087025

Lefevre, T., Ohm, J., Dabiré, K. R., Cohuet, A., Choisy, M., Thomas, M. B., et al. (2018). Transmission traits of malaria parasites within the mosquito: Genetic variation, phenotypic plasticity, and consequences for control. Evol. Appl. 11, 456-469. doi: 10.1111/eva.12571

Mitri, C., Bischoff, E., Takashima, E., Williams, M., Eiglmeier, K., Pain, A., et al. (2015). An evolution-based screen for genetic differentiation between anopheles sister taxa enriches for detection of functional immune factors. PLoS Pathog. 11, e1005306. doi: 10.1371/journal.ppat.1005306

Modrzynska, K., Pfander, C., Chappell, L., Yu, L., Suarez, C., Dundas, K., et al. (2017). A knockout screen of apiap2 genes reveals networks of interacting transcriptional regulators controlling the plasmodium life cycle. Cell Host Microbe 21, 11-22. doi: 10.1016/j.chom.2016.12.003

Molina-Cruz, A., DeJong, R. J., Charles, B., Gupta, L., Kumar, S., JaramilloGutierrez, G., et al. (2008). Reactive oxygen species modulate anopheles gambiae immunity against bacteria and plasmodium. J. Biol. Chem. 283, 3217-3223. doi: 10.1074/jbc.M705873200

Molina-Cruz, A., Garver, L. S., Alabaster, A., Bangiolo, L., Haile, A., Winikor, J., et al. (2013). The human malaria parasite pfs 47 gene mediates evasion of the mosquito immune system. Science 340, 984-987. doi: 10.1126/science.1235264

Molina-Cruz, A., Canepa, G. E., e Silva, T. L. A., Williams, A. E., Nagyal, S., Yenkoidiok-Douti, L., et al. (2020). Plasmodium falciparum evades immunity of anopheline mosquitoes by interacting with a pfs 47 midgut receptor. Proc. Natl. Acad. Sci. 117, 2597-2605. doi: 10.1073/pnas.1917042117

Moon, R. W., Taylor, C. J., Bex, C., Schepers, R., Goulding, D., Janse, C. J., et al. (2009). A cyclic gmp signalling module that regulates gliding motility in a malaria parasite. PLoS Pathog. 5, e1000599. doi: 10.1371/journal.ppat.1000599

Morrissette, N. S., and Sibley, L. D. (2002). Cytoskeleton of apicomplexan parasites. Microbiol. Mol. Biol. Rev. 66, 21-38. doi: 10.1128/MMBR.66.1.2138.2002

Muñoz, E. E., Hart, K. J., Walker, M. P., Kennedy, M. F., Shipley, M. M., and Lindner, S. E. (2017). Alba4 modulates its stage-specific interactions and specific mrna fates during plasmodium yoelii growth and transmission. Mol. Microbiol. 106, 266-284. doi: 10.1111/mmi.13762

Nikolaeva, D., Draper, S. J., and Biswas, S. (2015). Toward the development of effective transmission-blocking vaccines for malaria. Expert Rev. Vaccines 14, 653-680. doi: 10.1586/14760584.2015.993383
Nikolaeva, D., Illingworth, J. J., Miura, K., Alanine, D. G., Brian, I. J., Li, Y., et al. (2020). Functional characterization and comparison of plasmodium falciparum proteins as targets of transmission-blocking antibodies. Mol. Cell. Proteomics 19, 155-166. doi: 10.1074/mcp.RA117.000036

Osta, M. A., Christophides, G. K., and Kafatos, F. C. (2004). Effects of mosquito genes on plasmodium development. Science 303, 2030-2032. doi: 10.1126/ science.1091789

Otto, T. D., Wilinski, D., Assefa, S., Keane, T. M., Sarry, L. R., Böhme, U., et al. (2010). New insights into the blood-stage transcriptome of plasmodium falciparum using rna-seq. Mol. Microbiol. 76, 12-24. doi: 10.1111/j.13652958.2009.07026.x

Otto, T. D., Böhme, U., Jackson, A. P., Hunt, M., Franke-Fayard, B., Hoeijmakers, W. A., et al. (2014). A comprehensive evaluation of rodent malaria parasite genomes and gene expression. BMC Biol. 12, 86. doi: 10.1186/s12915-014-0086-0

Paskewitz, S. M., and Shi, L. (2005). Bypassing the midgut results in development of plasmodium berghei oocysts in a refractory strain of anopheles gambiae (diptera: Culicidae). J. Med. Entomol. 42, 712-715. doi: 10.1603/0022-2585 (2005)042[0712:BTMRID]2.0.CO;2

Patzewitz, E.-M., Guttery, D. S., Poulin, B., Ramakrishnan, C., Ferguson, D. J., Wall, R. J., et al. (2013). An ancient protein phosphatase, shlp1, is critical to microneme development in plasmodium ookinetes and parasite transmission. Cell Rep. 3, 622-629. doi: 10.1016/j.celrep.2013.01.032

Potocnjak, P., Yoshida, N., Nussenzweig, R. S., and Nussenzweig, V. (1980) Monovalent fragments (Fab) of monoclonal antibodies to a sporozoite surface antigen $(\mathrm{Pb} 44)$ protect mice against malarial infection. J. Exp. Med. 151 (6), 1504-1513.

Reid, A. J., Talman, A. M., Bennett, H. M., Gomes, A. R., Sanders, M. J., Illingworth, C. J., et al. (2018). Single-cell rna-seq reveals hidden transcriptional variation in malaria parasites. Elife 7, e33105. doi: 10.7554/eLife.33105

Rovira-Graells, N., Gupta, A. P., Planet, E., Crowley, V. M., Mok, S., de Pouplana, L. R., et al. (2012). Transcriptional variation in the malaria parasite plasmodium falciparum. Genome Res. 22, 925-938. doi: 10.1101/gr.129692.111

Santos, J. M., Lebrun, M., Daher, W., Soldati, D., and Dubremetz, J.-F. (2009). Apicomplexan cytoskeleton and motors: Key regulators in morphogenesis, cell division, transport and motility. Int. J. Parasitol. 39, 153-162. doi: 10.1016/ j.ijpara.2008.10.007

Siden-Kiamos, I., Ecker, A., Nybäck, S., Louis, C., Sinden, R. E., and Billker, O. (2006). Plasmodium berghei calcium-dependent protein kinase 3 is required for ookinete gliding motility and mosquito midgut invasion. Mol. Microbiol. 60, 1355-1363. doi: 10.1111/j.1365-2958.2006.05189.x

Simões, M. L., Dong, Y., Hammond, A., Hall, A., Crisanti, A., Nolan, T., et al. (2017). The anopheles fbn9 immune factor mediates plasmodium speciesspecific defense through transgenic fat body expression. Dev. Comp. Immunol. 67, 257-265. doi: 10.1016/j.dci.2016.09.012

Sinden, R. E. (1997). "Infection of mosquitoes with rodent malaria," in The molecular biology of insect disease vectors (Dordrecht: Springer), 67-91.

Smith, R. C., Vega-Rodríguez, J., and Jacobs-Lorena, M. (2014). The plasmodium bottleneck: Malaria parasite losses in the mosquito vector. Mem. Inst. Oswaldo Cruz 109, 644-661. doi: 10.1590/0074-0276130597

Tomas, A. M., Margos, G., Dimopoulos, G., Van Lin, L. H., de Koning-Ward, T. F., Sinha, R., et al. (2001). P25 and p28 proteins of the malaria ookinete surface have multiple and partially redundant functions. EMBO J. 20, 3975-3983. doi: $10.1093 /$ emboj/20.15.3975

Tremp, A. Z., and Dessens, J. T. (2011). Malaria imcl membrane skeleton proteins operate autonomously and participate in motility independently of cell shape. J. Biol. Chem. 286, 5383-5391. doi: 10.1074/jbc.M110.187195

Tremp, A. Z., Khater, E. I., and Dessens, J. T. (2008). Imclb is a putative membrane skeleton protein involved in cell shape, mechanical strength, motility, and infectivity of malaria ookinetes. J. Biol. Chem. 283, 2760427611. doi: 10.1074/jbc.M801302200

Turturice, B. A., Lamm, M. A., Tasch, J. J., Zalewski, A., Kooistra, R., Schroeter, E. H., et al. (2013). Expression of cytosolic peroxiredoxins in plasmodium berghei ookinetes is regulated by environmental factors in the mosquito bloodmeal. PLoS Pathog. 9, e1003136. doi: 10.1371/journal.ppat.1003136

Ukegbu, C. V., Akinosoglou, K. A., Christophides, G. K., and Vlachou, D. (2017). Plasmodium berghei pimms2 promotes ookinete invasion of the anopheles gambiae mosquito midgut. Infect. Immun. 85 (8), e00139-17. doi: 10.1128/ IAI.00139-17 
Ukegbu, C. V., Giorgalli, M., Tapanelli, S., Rona, L. D., Jaye, A., Wyer, C., et al. (2020). Pimms43 is required for malaria parasite immune evasion and sporogonic development in the mosquito vector. Proc. Natl. Acad. Sci. 117, 7363-7373. doi: 10.1073/pnas.1919709117

Volkmann, K., Pfander, C., Burstroem, C., Ahras, M., Goulding, D., Rayner, J. C., et al. (2012). The alveolin imclh is required for normal ookinete and sporozoite motility behaviour and host colonisation in plasmodium berghei. PLoS One 7, e41409. doi: 10.1371/journal.pone.0041409

Waters, A. P. (2016). Epigenetic roulette in blood stream plasmodium: Gambling on sex. PLoS Pathog. 12, e1005353. doi: 10.1371/journal.ppat.1005353

White, B. J., Lawniczak, M. K., Cheng, C., Coulibaly, M. B., Wilson, M. D., Sagnon, N. F., et al. (2011). Adaptive divergence between incipient species of anopheles gambiae increases resistance to plasmodium. Proc. Natl. Acad. Sci. 108, 244249. doi: $10.1073 /$ pnas. 1013648108

Winger, L. A., Tirawanchai, N., Nicholas, J., Carter, H. E., Smith, J. E., and Sinden, R. E. (1988) Ookinete antigens of Plasmodium berghei. Appearance on the zygote surface of an Mr $21 \mathrm{kD}$ determinant identified by transmission-blocking monoclonal antibodies. Parasite Immunol. 10 (2), 193-207. doi: 10.1111/ j.1365-3024.1988.tb00214.x
Yeoh, L. M., Goodman, C. D., Mollard, V., McFadden, G. I., and Ralph, S. A. (2017). Comparative transcriptomics of female and male gametocytes in plasmodium berghei and the evolution of sex in alveolates. BMC Genomics 18, 734. doi: 10.1186/s12864-017-4100-0

Zheng, W., Kou, X., Du, Y., Liu, F., Yu, C., Tsuboi, T., et al. (2016). Identification of three ookinete-specific genes and evaluation of their transmission-blocking potentials in plasmodium berghei. Vaccine 34, 2570-2578. doi: 10.1016/ j.vaccine.2016.04.011

Conflict of Interest: The authors declare that the research was conducted in the absence of any commercial or financial relationships that could be construed as a potential conflict of interest.

Copyright (C) 2021 Ukegbu, Christophides and Vlachou. This is an open-access article distributed under the terms of the Creative Commons Attribution License (CC BY). The use, distribution or reproduction in other forums is permitted, provided the original author(s) and the copyright owner(s) are credited and that the original publication in this journal is cited, in accordance with accepted academic practice. No use, distribution or reproduction is permitted which does not comply with these terms. 